\title{
Potential Privilege of Maltodextrin- $\alpha$-Tocopherol Nano-Micelles in Seizing Tacrolimus Renal Toxicity, Managing Rheumatoid Arthritis and Accelerating Bone Regeneration
}

\begin{abstract}
Hala M Helal, (D) 'Wael M Samy,' Elbadawy A Kamoun, ${ }^{2,3}$ Esmail M El-Fakharany, (ID ${ }^{4}$ Doaa A

Abdelmonsif, (iD ${ }^{5,6}$ Rania G Aly, ${ }^{7}$ Sana

M Mortada,' Marwa A Sallam'

'Department of Industrial Pharmacy, Faculty of Pharmacy, Alexandria University, Alexandria,

2152 I, Egypt; ${ }^{2}$ Polymeric Materials Research

Dep., Advanced Technology and New Materials

Research Institute (ATNMRI), City of Scientific

Research and Technological Applications

(SRTA-City), New Borg Al-Arab City,

Alexandria, 21934, Egypt; ${ }^{3}$ Nanotechnology

Research Center (NTRC), The British

University in Egypt (BUE), El- Sherouk City,

Cairo, I 1837, Egypt; ${ }^{4}$ Proteins Research Dep.,

Genetic Engineering and Biotechnology

Research Institute (GEBRI), City of Scientific

Research and Technological Applications

(SRTA-City), New Borg Al-Arab City,

Alexandria, 21934 , Egypt; ${ }^{5}$ Department of

Medical Biochemistry, Faculty of Medicine,

Alexandria University, Alexandria, 2I52I,

Egypt; ${ }^{6}$ Center of Excellence for Research in

Regenerative Medicine and Applications

(CERRMA), Faculty of Medicine, Alexandria

University, Alexandria, 2152I, Egypt;

${ }^{7}$ Department of Surgical Pathology, Faculty of

Medicine, Alexandria University, Alexandria,

21521, Egypt
\end{abstract}

Correspondence: Marwa A Sallam

Department of Industrial Pharmacy,

Faculty of Pharmacy, Alexandria

University, Alexandria, 21521, Egypt

Tel +2034868256

Fax +2034871668

Email marwa.sallam@alexu.edu.eg

Elbadawy A Kamoun

Polymeric Materials Research Dep.,

Advanced Technology and New Materials

Research Institute (ATNMRI), City of

Scientific Research and Technological

Applications (SRTA-City), New Borg

Al-Arab City, Alexandria, 21934 , Egypt

Tel +20 1283320302

Email e-b.kamoun@tu-bs.de
Background: Tacrolimus (TAC) is a powerful immunosuppressive agent whose therapeutic applicability is confined owing to its systemic side effects.

Objective: Herein, we harnessed a natural polymer based bioconjugate composed of maltodextrin and $\alpha$-tocopherol (MD- $\alpha$-TOC) to encapsulate TAC as an attempt to overcome its biological limitations while enhancing its therapeutic anti-rheumatic efficacy.

Methods: The designed TAC loaded maltodextrin- $\alpha$-tocopherol nano-micelles (TAC@MD$\alpha$-TOC) were assessed for their physical properties, safety, toxicological behavior, their ability to combat arthritis and assist bone/cartilage formation.

Results: In vitro cell viability assay revealed enhanced safety profile of optimized TAC@MD- $\alpha$-TOC with 1.6- to 2-fold increase in Vero cells viability compared with free TAC. Subacute toxicity study demonstrated a diminished nephro- and hepato-toxicity accompanied with optimized TAC@MD- $\alpha$-TOC. TAC@MD- $\alpha$-TOC also showed significantly enhanced anti-arthritic activity compared with free TAC, as reflected by improved clinical scores and decreased IL- 6 and TNF- $\alpha$ levels in serum and synovial fluids. Unique bone formation criteria were proved with TAC@MD- $\alpha$-TOC by elevated serum and synovial fluid levels of osteocalcin and osteopontin mRNA and proteins expression. Chondrogenic differentiation abilities of TAC@MD- $\alpha$-TOC were proved by increased serum and synovial fluid levels of SOX9 mRNA and protein expression.

Conclusion: Overall, our designed bioconjugate micelles offered an excellent approach for improved TAC safety profile with enhanced anti-arthritic activity and unique bone formation characteristics.

Keywords: polymeric micelles, vitamin E, osteopontin, SOX9, rheumatoid

\section{Introduction}

Rheumatoid arthritis (RA) is a chronic inflammatory malady where autoimmunity plays a vital role in its appearance and progression. It is induced by different genetic, environmental, infectious, sexual and hormonal factors leading to pain, stiffness, swelling, erosions of the cartilage, and bone joint deformity. ${ }^{1,2}$ Strategies implemented in the management of RA are surgery and use of different classes of medicines, the most important of which in clinical practice are disease modifying anti-rheumatic drugs (DMARDs). However, their long-term systemic administration leads to obvious systemic toxicity. ${ }^{3}$ 


\section{Graphical Abstract}

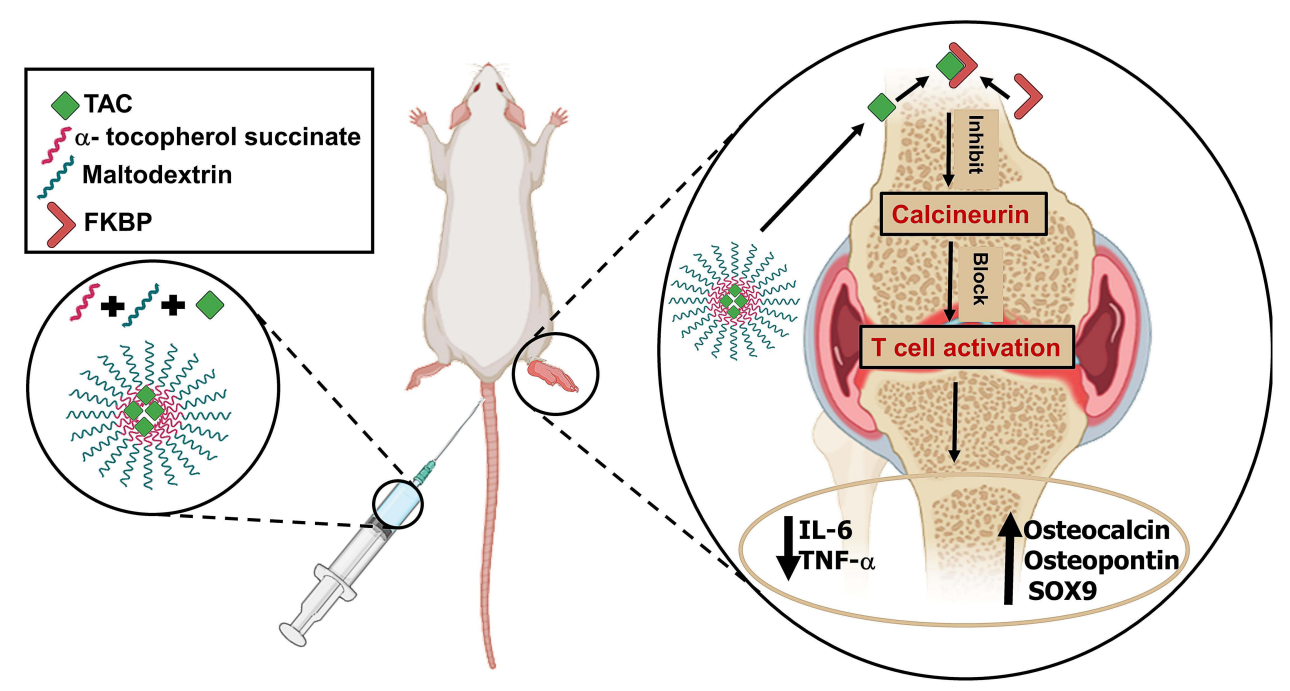

Tacrolimus (TAC, FK 506) is a lipophilic antibiotic with 23-member lactone structure that is isolated from Streptomyces tsukubaensis with immunomodulatory and anti-inflammatory effects. It belongs to the disease modifying anti-rheumatic drugs (DMARD), exerting its immunosuppression mainly by interposing with the activation of T-cells. TAC links to intracellular proteins termed immunophilins; FK506 binding proteins (FKBP), after entering T-cells. This combined complex works on inhibiting calcineurin phosphatase which is a transcription factor required for the expression of cytokine genes in T-cells, resulting in a complete block of production of T-cell-derived cytokines such as interleukin (IL-2) and interferon- $\gamma \cdot{ }^{4}$ Tacrolimus is a BCS Class II drug that exhibits very poor water solubility $(\log P 3.96 \pm 0.83)$. It has a high molecular weight $(804 \mathrm{~g} / \mathrm{mol})$ and causes severe side effects most important of which is nephrotoxicity, limiting its clinical use. ${ }^{4,5}$

Amphiphilic polymeric nano-micelles have gained global attention due to their high loading capacity of poorly water-soluble drugs. ${ }^{6}$ Their structure is formed of block copolymers or graft copolymers that undergo assembly in aqueous medium owing to their amphiphilic characters. There is a vast interest in tailored nanomicelles, especially those of natural origin, i.e. polysaccharides, as they enhance water solubility with decreased adverse effects of active pharmaceutical ingredients. ${ }^{7}$ Polymeric micelles provide a controlled drug release allowing long circulation time for poorly soluble drugs, thus offering suitability for intravenously administered formulations. Their size distribution provides the merit of combining the prevention of premature elimination by glomerular filtration and the passage through certain blood vessels depending on their small size. Consequently, tailored polymeric micelles can allow longer mean residence time of the drug in the circulation, enhanced bioavailability, reduced submitted dose and decreased non-specific organ toxicity. ${ }^{8}$ Accordingly, they appear to possess the potential to handle various TAC obstacles that limit its systemic applications.

In this study, we designed amphiphilic micelles based on maltodextrin- $\alpha$-tocopherol bioconjugate (MD- $\alpha$-TOC) encapsulating TAC, in an attempt to seize TAC toxicity, manage RA, and enhance bone formation characteristics (Grafical abstract). Previous studies focused on evaluating the effect of TAC loaded-nanocarriers for RA management; however, the capability of promoting bone formation has not been previously reported. ${ }^{9-11}$

Herein, we aim to tackle the limitations of TAC, enhance its anti-arthritic properties, and to introduce new impacts on bone formation process by evaluating the related biomarkers during RA condition.

\section{Materials and Methods \\ Materials}

Materials are described in Supporting Information. 
A

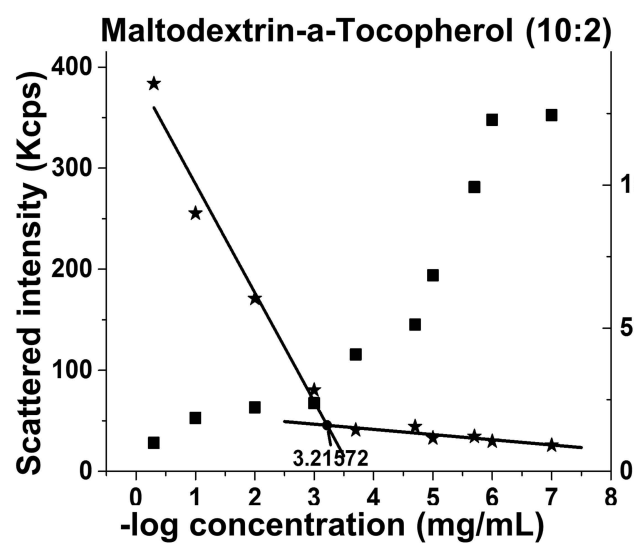

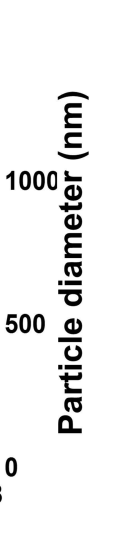

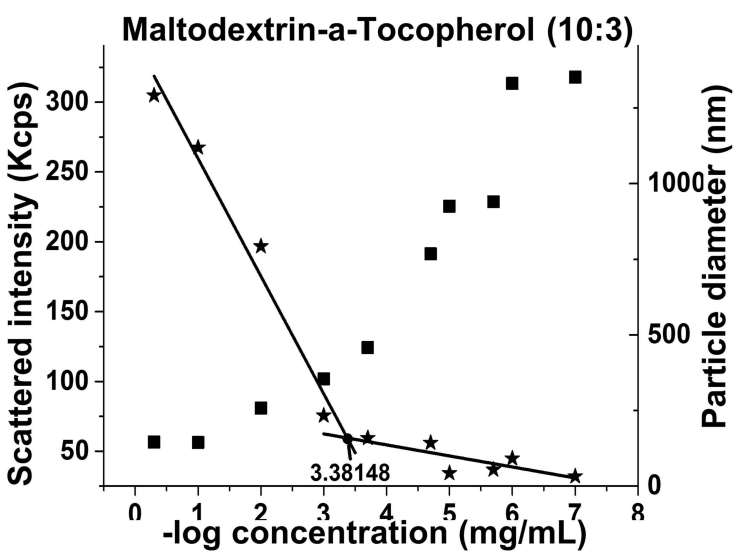

B
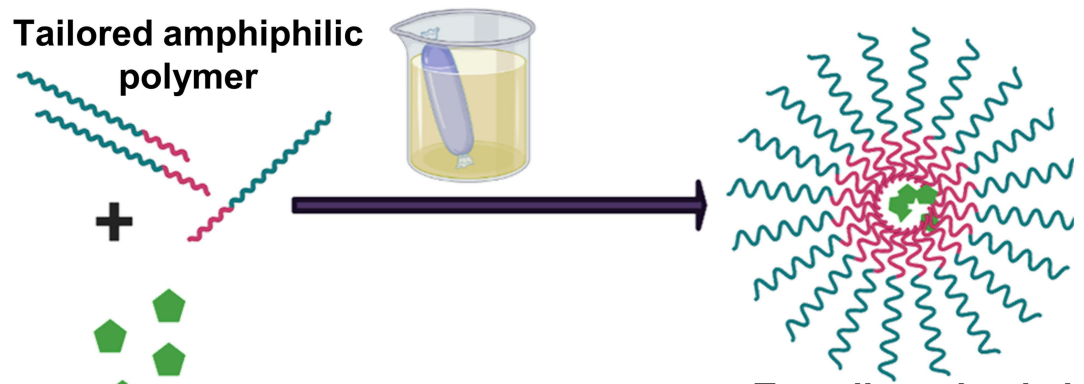

Tacrolimus

Tacrolimus loaded polymeric micelles

C
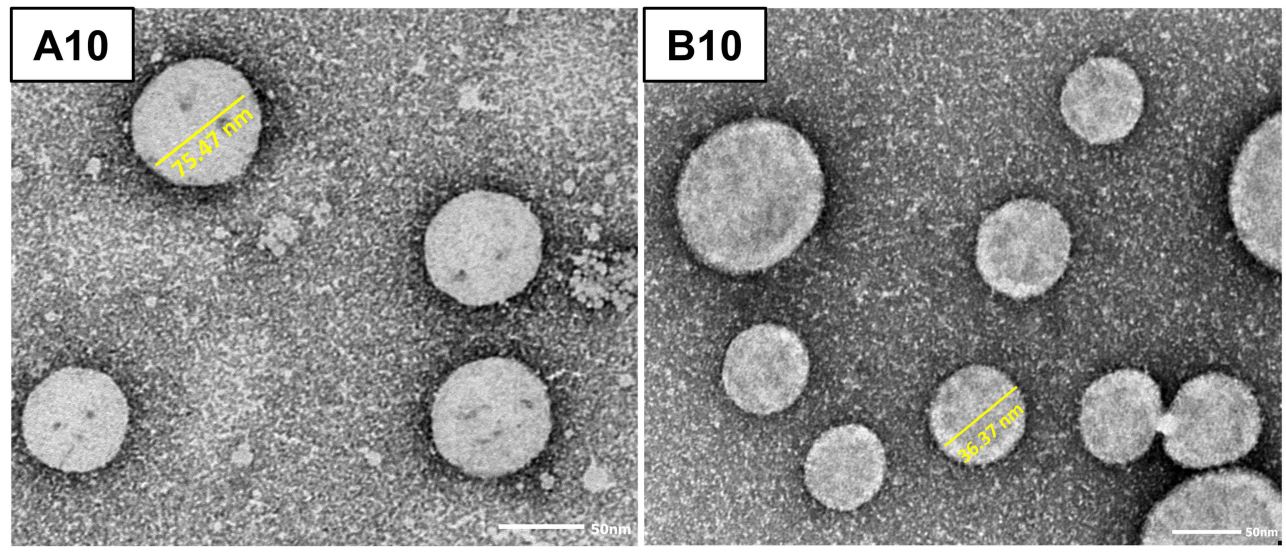

D

Size distribution by intensity (A10)

Size distribution by intensity (B10)
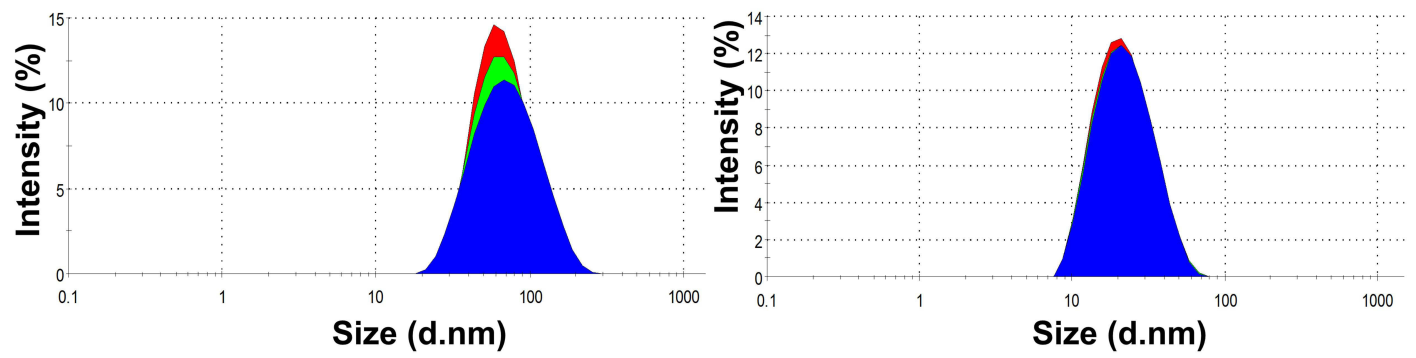

Figure I (A) Determination of CMC by DLS: A comparative plot of intensity of scattered light (left axis, $\star$ ) and hydrodynamic diameter (right axis, $\mathbf{\square}$ ) as a function different polymer concentrations (B) Schematic presentation for the preparation of TAC loaded polymeric micelles (C) TEM micrograph showing morphology of AI $\mathbf{0}$ and B I $\mathbf{0}$ with core-shell structure and (D) particle size distribution diagram of AlO and B I 0. 


\section{Methodology}

Preparation \& Characterization of TAC-Loaded MD$\alpha$-TOC Polymeric Micelles

MD- $\alpha$-TOC bioconjugates were prepared with two different molar ratios according to the synthetic procedure described in Supporting Information. (Figure S1) ${ }^{12}$ TAC-loaded micelles (TAC@MD- $\alpha-\mathrm{TOC}$ ) were prepared by direct dialysis method. ${ }^{13}$ Twenty-five mg of the synthesized MD- $\alpha$-TOC conjugates $(10: 2,10: 3)$ were dissolved separately in $2 \mathrm{~mL}$ DMSO along with varied amounts of TAC for optimization. The dispersions obtained were packed into dialysis tubes (Spectra/Por 6) with a MWCO $1 \mathrm{KDa}$ then sealed. Dialysis was performed for $24 \mathrm{~h}$ against $\left(\mathrm{H}_{2} \mathrm{O}\right.$ : DMSO, 50:50) for the first $4 \mathrm{~h}$, and then completed against $100 \%$ distilled water which was exchanged every $4 \mathrm{~h}$ for the rest of the $24 \mathrm{~h}$. The prepared micellar solutions were then filtered using $0.45 \mu \mathrm{m}$ syringe filters before subsequent characterization to remove unloaded drug. The physicochemical characterization of TACloaded MD- $\alpha$-TOC nano-micelles is described in Supporting Information.

\section{$\mathrm{pH}$-Dependent Drug Release of TAC from TAC- Loaded MD- $\alpha$-TOC Nano-Micelles}

In vitro release of TAC was assessed using dialysis tubes method in USP XXIII type-II dissolution apparatus as described in Supporting Information. ${ }^{14}$

\section{In vitro Stability of TAC-Loaded MD- $\alpha$-TOC Nano- Micelles}

\section{Stability Upon Storage Conditions}

Stability studies were carried out over a period of 3 months. Micelles were kept in glass vials sealed with plastic caps and were stored in the fridge at a temperature of $4-8^{\circ} \mathrm{C}$. The formulations were monitored for physical changes, particle size, ZP, and PDI; as well as the chemical stability of encapsulated TAC. ${ }^{15}$

\section{In vitro Serum Stability}

To mimic blood circulation conditions, samples of TAC@MD- $\alpha$-TOC (3 mg polymer $/ \mathrm{mL}$ ) were incubated with $10 \%$ of fetal bovine serum at a ratio of (1:1) for $6 \mathrm{~h}$ at $37^{\circ} \mathrm{C}$ under gentle stirring. Samples were taken at different time intervals $(0,1,2,4,6 \mathrm{~h})$ and assessed for changes in micellar particle size. ${ }^{16}$

Micellar Stability Upon Dilution

Specified volume of each TAC@MD- $\alpha$-TOC micellar dispersion (A10 and B10) was mixed with appropriate volume of distilled water to be diluted 10,50, 100 times. Any change in particle size and PDI was monitored by dynamic light scattering (DLS) technique. ${ }^{17}$

\section{In vitro and in vivo Toxicological Assessment of TAC@ MD- $\alpha$-TOC Nano-Micelles}

\section{Hemolytic Activity Assay}

Free TAC, A10 and B10 TAC-loaded nano-micelles at a concentration of $3 \mathrm{mg} \mathrm{TAC} / \mathrm{mL}$ in $0.9 \% \mathrm{NaCl}$ saline solution were prepared for hemolysis assay. ${ }^{18}$ The hemolytic activity was investigated using rat red blood cells (RBCs) separated from plasma washed three times with normal saline. Details are provided in Supporting Information.

\section{In vitro Cytotoxicity Test}

The effect of free TAC, A10 and B10 TAC@, MD- $\alpha$-TOC nano-micelles on viability of Vero cells (kidney epithelial cells of African green monkey) and peripheral blood mononuclear cells PBMCs was evaluated using MTT cell viability assay ${ }^{19}$ (details in SI).

\section{In vivo Sub-Acute Toxicity Study}

Twenty adult-female Wistar rats (6 weeks old, weighing $170-190 \mathrm{~g}$ ) were kept in standard cages at $25^{\circ} \mathrm{C}$ and with a $12 \mathrm{~h}$-light/12h-dark cycle in the animal house of the Faculty of Medicine, Alexandria University. Rats were fed standard chow and water ad libitum during the study duration and they were allowed to adapt to their environment for 10 days before starting the experiments. All animal experiments obeyed the ARRIVE guidelines and were performed in accordance with the UK Animals (Scientific Procedures) Act, 1986 and associated guidelines, EU Directive 2010/63/EU for animal experiments. Additionally, all procedures involved in animal experiments were carried out according to International Council for Laboratory Animal Science (ICLAS)Alexandria University guidelines (06-2020-2-10-2-69) and were approved by the institutional ethical committee. Rats were randomly divided into four groups ( 5 rats per group) receiving $0.5 \mathrm{~mL}$ IV injection of the test solution via the tail vein as follows: group 1 (i.e. negative controls) received $0.5 \mathrm{~mL}$ saline, group 2 received $0.5 \mathrm{~mL}(1 \mathrm{mg} / \mathrm{kg})$ free TAC, group 3 were administered $0.5 \mathrm{~mL}$ of saline dispersion of A10 (corresponding to dose of TAC of $1 \mathrm{mg} / \mathrm{kg}$ loaded in MD- $\alpha$-TOC (10:2)) and group 4 that received $0.5 \mathrm{~mL}$ of saline dispersion of $\mathbf{B 1 0}$ (corresponding to dose of TAC of $1 \mathrm{mg} / \mathrm{kg}$ loaded in MD- $\alpha$-TOC (10:3)). The doses were prepared under aseptic conditions to assure their sterility 
before IV injection and they were given every 2 days for a duration of 14 days. ${ }^{20,21}$

\section{Animal Observations}

Clinical signs of toxicity and mortalities were observed during the whole study. Body weights were recorded at the beginning of the experiment and once weekly afterwards to ensure safety of test samples. Animals were sacrificed $24 \mathrm{~h}$ after the last dose given on day 14. After sacrifice, gross examination of the animals' organs was performed, and their livers and kidneys were extracted for histopathological observations of any significant toxicological signs.

\section{Biochemical Tests}

For hematological assessment, a blood sample was collected from each rat's retro-orbital plexus. Hematological parameters including RBC, WBC, platelets and differential leukocyte counts, hemoglobin concentration $(\mathrm{Hb})$, hematocrit (HCT), red cell distribution width (RDW) and red cell indices [i.e. mean corpuscular volume (MCV), mean corpuscular hemoglobin $(\mathrm{MCH})$ and mean corpuscular hemoglobin concentration (MCHC)] were analyzed using an Advia 120 Hematology Analyzer (Siemens, Germany). Another blood sample was collected for assessment of serum biochemical parameters of liver and kidney function. Possible hepatotoxicity was assessed spectrophotometrically using ALT and AST Spectrum assay kits. Also, serum levels of urea and creatinine were evaluated to detect any potential nephrotoxicity.

\section{Histopathological Study}

Liver and kidney samples were fixed in $10 \%$ formalin, soaked in paraffin blocks, then were cut, positioned on glass slides and stained with H\&E. Specimens were examined and photos were taken using a light microscope.

\section{Adjuvant-Induced Arthritis (AIA) Model in Rats}

Arthritis was induced in female Wistar rats (5-6 weeks old weighing $140-160 \mathrm{~g}$ ) by subplantar injection of $100 \mu \mathrm{L}$ of complete Freund's adjuvant CFA $(1 \mathrm{mg} / \mathrm{mL}$ heat killed and dried Mycobacterium tuberculosis in $0.85 \mathrm{~mL}$ mineral oil, $0.15 \mathrm{~mL}$ mannide mono-oleate) in the left hind paw. Additional dose (100 $\mu \mathrm{L} \mathrm{CFA)} \mathrm{was} \mathrm{given} \mathrm{to} \mathrm{all} \mathrm{animals}$ on the 7th and 14th day of the primary dose. On the 15th day of primary dose, the treatment was started. Animals were divided into 7 groups $(\mathrm{n}=5)$, treated as following: group 1: received neither CFA nor treatment to act as negative control; group 2: received saline and
Table I Primers Sequences Used for qRT-PCR in AIA Study

\begin{tabular}{|l|l|l|}
\hline Target & Sequence & $\begin{array}{l}\text { Accession } \\
\text { Number }\end{array}$ \\
\hline Osteocalcin & $\begin{array}{l}\text { AAG GTG GTG AAT AGA CTC } \\
\text { CG (Forward) } \\
\text { AAA CGG TGG TGC CAT AGA } \\
\text { TG (Reverse) }\end{array}$ & NM_0I34I4.I \\
\hline Osteopontin & $\begin{array}{l}\text { CTC AGA GGA GAA GGC GCA } \\
\text { TTG (Forward) } \\
\text { TCT CTG CAT GGT CTC CGT } \\
\text { CGT (Reverse) }\end{array}$ & NM_0I288I.2 \\
\hline SOX9 & $\begin{array}{l}\text { AGGAAGCTGGCAGACCAGTA } \\
\text { (Forward) } \\
\text { CGAAGGGTCTCTTCTCGCT } \\
\text { (Reverse) }\end{array}$ & NM_080403.I \\
\hline GAPDH & $\begin{array}{l}\text { TGCCACTCAGAAGACTGTGG } \\
\text { (Forward) } \\
\text { TTCAGCTCTGGGATGACCTT } \\
\text { (Reverse) }\end{array}$ & NM_0I7008.4 \\
\hline
\end{tabular}

represented positive group for AIA arthritic model; group 3: received free TAC (1 $\mathrm{mg} / \mathrm{kg})$; group 4: received A10 corresponding to (1 mg TAC/Kg); group 5: received $\mathbf{A 1 0}$ blank micelles; group 6: received $\mathbf{B 1 0}$ corresponding to (1 mg TAC/Kg); group 7: received B10 blank micelles. All rats received treatment via IV injection through the tail vein every 2 days. Body weights of each group were monitored during the study and mortality rates were recorded, while paw thicknesses of rats were measured thrice a week by Vernier's caliper. Left hind paws were carefully given arthritis scores based on macroscopic observations. Arthritis score was given as: $0=$ no signs of arthritis, $1=$ swelling and/or redness of one digit, 2 = two joints involved, $3=$ more than two joints involved, and $4=$ severe arthritis of the entire paw and all digits. The arthritis severity score for each group was calculated by adding the scores of each paw in the individual rat. ${ }^{3,22}$ On day 30 of the study, synovial fluids and blood samples were collected from the animals for determination of inflammatory, bone formation and chondrogenic differentiation biomarkers, and hind paws were collected for histological examination.

\section{Determination of Inflammatory Biomarkers}

Synovial fluid and serum levels of TNF- $\alpha$ and IL-6 were determined using commercially available rat Quantikine ELISA kits according to R\&D instructions. All samples were analyzed in duplicate and results were expressed in $\mathrm{pg} / \mathrm{mL}$. 


\section{Determination of Bone Formation Biomarkers}

Synovial fluid and serum level of osteocalcin and osteopontin proteins were determined following MyBioSource ratspecific ELISA kits instructions. All samples were analyzed in duplicate and results were expressed in $\mathrm{mg} / \mathrm{L}$. The relative expression of synovial fluid osteocalcin and osteopontin mRNA were determined by quantitative reversetranscription PCR (qRT-PCR). For that purpose, total RNA was extracted from synovial fluid samples under strict RNAse-free conditions and following RN easy Mini Kit protocol. The quantity and quality of extracted RNA were judged by NanoDrop 1000 Spectrophotometer (Thermo Scientific, USA). Afterwards, RNA was reverse transcribed using Superscript II reverse transcriptase kit. The qRT-PCR was then performed in duplicate ( $25 \mu \mathrm{L}$ reaction volume) in StepOne real-time PCR system (Applied Biosystems, USA) using $1 \mathrm{XSybr}^{\circledR}$ Green PCR master mix. This was followed by melting curve analysis to ascertain the identity of the PCR product. StepOne ${ }^{\mathrm{TM}}$ Software v2.3 was used for data analysis where results were normalized to glyceraldehyde-3-phosphate dehydrogenase (GAPDH) mRNA levels. ${ }^{23}$ The results were then expressed as fold change of treated groups relative to negative control group. The specific primer pairs for osteocalcin, osteopontin and GAPDH are listed in Table $1 .{ }^{24}$

\section{Determination of Chondrogenic Differentiation Biomarkers} Synovial fluid and serum levels of chondrogenic differentiation protein; SOX9 (ng/mL) were measured according to rat specific MyBioSource ELISA kit instructions. Additionally, synovial fluid SOX9 mRNA expression was determined by qRT-PCR as described above using specific SOX9 primers (Table 1). ${ }^{25}$

\section{Histopathological Examination}

Hematoxylin and Eosin (H\&E) Staining

Histopathological examinations of separated hind paws specimens were performed. Paws were fixed in $10 \%$

Table 2 Physico-Chemical Characterization of MaltodextrinA-Tocopherol Conjugates

\begin{tabular}{|c|c|c|c|c|}
\hline$\#$ & $\begin{array}{c}\text { Composition } \\
\text { Ratio Of } \\
\text { Conjugate (MD } \\
\text { Repeating Units: } \\
\boldsymbol{\alpha - T O C )}\end{array}$ & $\begin{array}{c}\text { Molecular } \\
\text { Weight } \\
\text { ('H-NMR) } \\
(\mathbf{D a})\end{array}$ & $\begin{array}{c}\text { DD\% } \\
\text { ('H-NMR) }\end{array}$ & $\begin{array}{c}\text { CMC } \\
(\mathrm{mg} / \mathrm{mL})\end{array}$ \\
\hline A & $10: 2$ & 5566.989 & 7.90 & $6.08 \times 10^{-4}$ \\
$B$ & $10: 3$ & 5902.481 & 10.28 & $4.15 \times 10^{-4}$ \\
\hline
\end{tabular}

formalin for $24 \mathrm{~h}$ and then decalcified in $10 \%$ formic acid. Sections were cut at $4 \mu \mathrm{m}$ thicknesses, stained with $\mathrm{H} \& \mathrm{E}$ and viewed under a light microscope.

\section{Alcian Blue Staining for Proteoglycan}

Staining with Alcian blue was conducted to indicate cartilage damage in joints. Joints sections of $5 \mu \mathrm{m}$ were cut from $10 \%$ formalin fixed, paraffin-embedded tissue blocks and mounted on poly-L-lysine coated microscope slides. Slides were then evaluated under light microscope. ${ }^{21}$

\section{Statistical Analysis}

Data were analyzed using IBM SPSS software package version 20.0. (Armonk, NY: IBM Corp). Quantitative data were described using range (minimum and maximum), mean, standard deviation and standard error. Significance of the obtained results was judged at the 5\% level. The results of the in vivo study were analyzed using F-test (ANOVA) with Post Hoc test (Tukey) for pairwise comparisons.

\section{Results and Discussion}

Tailored MD- $\alpha$-TOC bioconjugates were synthesized following a simple carbodiimide method. ${ }^{12,26}$ Chemical entities and physicochemical properties of maltodextrin- $\alpha$ tocopherol were investigated and confirmed by several techniques. ${ }^{12}$ Noteworthy, synthesized biopolymers were obtained by utilizing two natural substrates whose merits were numerous owing to their biocompatibility, low immunogenicity, well-known structure and good tolerability within the human body. ${ }^{27}$ Instrumental characterization of bioconjugates by ${ }^{1} \mathrm{H}-\mathrm{NMR}$ (Figure S2, Supporting Information), FT-IR analyses performed previously $^{12}$ revealed the successful preparation of MD- $\alpha$-TOC bioconjugates by esterification reaction with formation of abundant ester bond at $v 1740 \mathrm{~cm}^{-1}(\mathrm{C}=\mathrm{O}$ stretching of esters $)$ at both molar ratios (10:2 and 10:3), suggesting successful grafting of $\alpha$-tocopherol onto maltodextrin. Some physicochemical properties of obtained MD- $\alpha$-TOC bio-conjugate polymers are shown in Table 2.

\section{Determination of Critical Micelle Concentration (CMC)}

CMCs were determined by simple DLS technique. ${ }^{28}$ This method was previously reported as a sensitive and convenient method when compared with fluorescence spectroscopy especially while determining low CMCs. ${ }^{28}$ Moreover, DLS technique offered the determination of hydrodynamic 

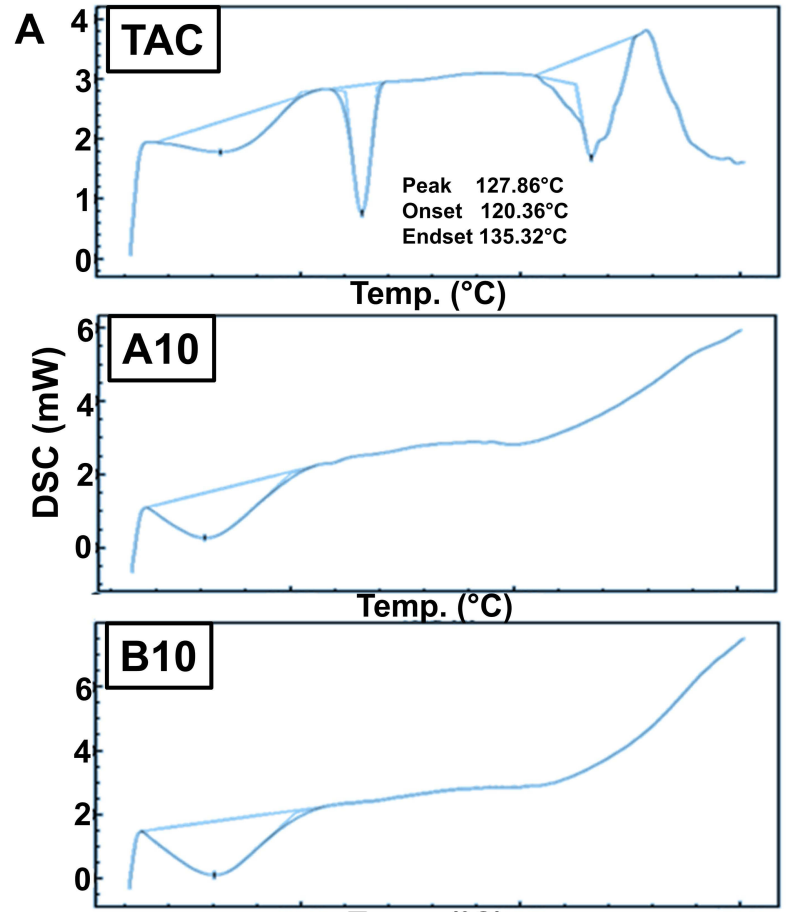

Temp. $\left({ }^{\circ} \mathrm{C}\right)$
B

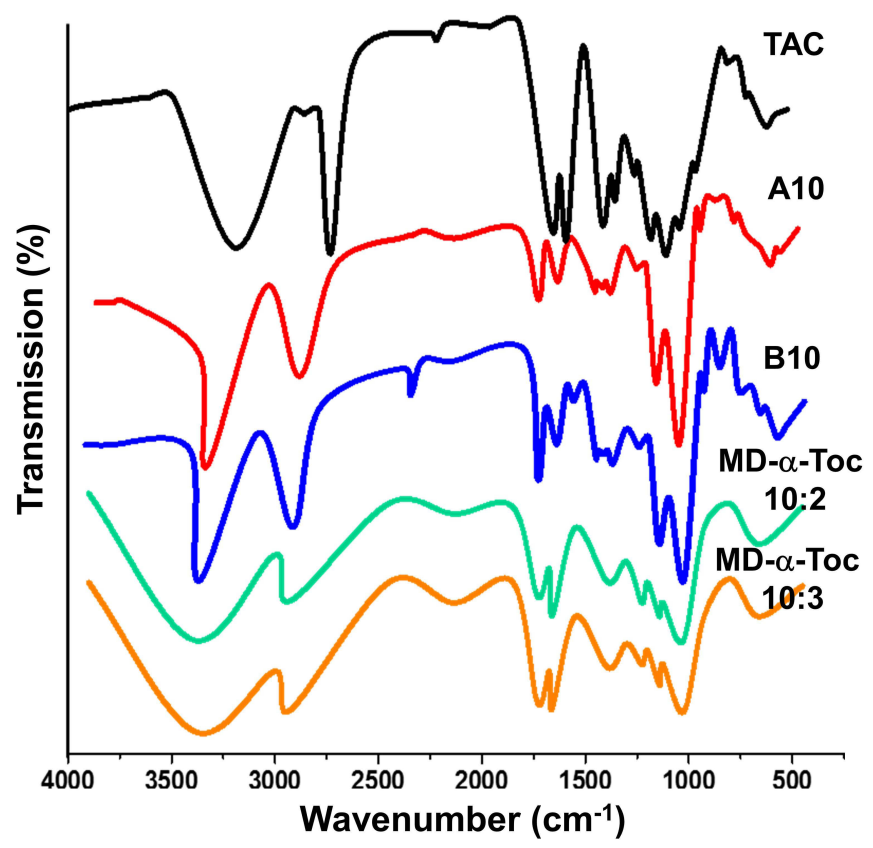

Figure 2 Solid state characterization (A) DSC thermograms of TAC, AI0 and BI0. (B) FT-IR spectra of TAC, AI0, B I0, maltodextrin- $\alpha$-tocopherol (I0:2) and maltodextrin- $\alpha$-tocopherol (10:3).

diameter of formed micelles besides CMC values using the same sample solutions (Figure 1A). CMCs values of synthesized conjugates shown in Table 2, seemed to be obviously very low which might enhance the stability of prepared nanomicellar structures upon dilution with physiological fluids, thus overcoming the main obstacle facing nano-micelles as drug delivery systems. ${ }^{12}$

\section{Preparation and Characterization of TAC-Loaded MD- $\alpha$-TOC Nano-Micelles} PS, Zeta Potential, PDI, EE and DL

Direct dialysis method was chosen over other techniques for preparation of nano-micelles, as shown in Figure 1B. This method granted an efficient dispersion of the drug during the micelle formation as both TAC and polymer were dissolved in DMSO. Upon slow displacement of DMSO by water during the dialysis step, TAC is incorporated within the hydrophobic core of the micelles instead of precipitation in water. For this reason, the dialysis stage was performed on two steps to avoid sudden precipitation of TAC in pure water. ${ }^{13}$
Results of particle size (PS), zeta-potential, PDI, EE and DL are represented in Table 3 . An increase in polymer concentration decreased the particle size. This effect could be owed to the micellization phenomenon where at low polymer concentration (above the $\mathrm{CMC}$ ), the hydrophobic portion of the copolymer avoided contact with the aqueous media thus forming core-shell micellar structures. However, the quantity of solvent lying inside the core of micellar structures would increase their particle size. Further increase in polymer concentration would cause release of the solvent from the core thus decreasing micellar PS (Table 3). ${ }^{29}$ Besides that, drug loading also influenced PS where highest drug loading was accompanied by the largest PS due to the presence of higher amounts of drug within the hydrophobic core. Degree of derivatization (DD) also affected micellar PS where a slight increase in DD resulted in decrease in PS values. This effect was consistent with results obtained by Wei et al. ${ }^{30}$ which was attributed to the fact that higher DD gives a densely packed hydrophobic core because of enhanced hydrophobic interaction. 
Table 3 Effect of DD Value, Polymeric Composition Ratio of Maltodextrin- $\alpha$-Tocopherol Nano-micelles and TAC Ratios on the Following: Particle Size, Zeta Potential, PDI, and EE\%

\begin{tabular}{|l|c|c|c|c|c|c|c|}
\hline$\#$ & Polymer & Polymer: Drug (wt:wt) & Particle Size (d.nm) & Zeta Potential (mv) & PDI & EE (w/w) \% & DL (w/w) \% \\
\hline$A I$ & $10: 2$ & $I: I$ & $194.5 \pm 12.75$ & $-13.21 \pm 1.3$ & $0.518 \pm 0.065$ & $31.47 \% \pm 2.97$ & $15.73 \% \pm 1.39$ \\
$B I$ & $10: 3$ & $I: I$ & $144.4 \pm 7.60$ & $-11.2 \pm 2.32$ & $0.675 \pm 0.024$ & $34.51 \% \pm 3.91$ & $17.25 \% \pm 0.73$ \\
$A 5$ & $10: 2$ & $5: 1$ & $134.1 \pm 2.42$ & $-19.9 \pm 2.43$ & $0.218 \pm 0.008$ & $47.46 \% \pm 8.26$ & $7.91 \% \pm 0.91$ \\
$B 5$ & $10: 3$ & $5: 1$ & $97.7 \pm 7.16$ & $-16.2 \pm 3.95$ & $0.379 \pm 0.028$ & $53.93 \% \pm 5.33$ & $8.98 \% \pm 0.36$ \\
$A 10$ & $10: 2$ & $10: 1$ & $74.78 \pm 0.14$ & $-22.5 \pm 6.05$ & $0.122 \pm 0.004$ & $94.85 \% \pm 4.95$ & $8.55 \% \pm 0.12$ \\
$B 10$ & $10: 3$ & $10: 1$ & $43.53 \pm 0.33$ & $-20.3 \pm 6.66$ & $0.287 \pm 0.004$ & $97.95 \% \pm 8.37$ & $8.90 \% \pm 0.87$ \\
$A 20$ & $10: 2$ & $20: 1$ & $68.03 \pm 0.94$ & $-24.6 \pm 2.15$ & $0.298 \pm 0.016$ & $95.01 \% \pm 5.19$ & $8.71 \% \pm 0.17$ \\
$B 20$ & $10: 3$ & $20: 1$ & $39.08 \pm 0.24$ & $-22.1 \pm 8.46$ & $0.225 \pm 0.003$ & $96.43 \% \pm 3.52$ & $8.78 \% \pm 0.21$ \\
\hline
\end{tabular}

Zeta potential was also influenced by polymer concentration and DD where increasing polymer concentration increased the surface negative charge of prepared micelles due to abundant surface $\mathrm{OH}$ - groups correlated to maltodextrin molecules. It was also noticed that the polymer with the higher DD value showed decrease in surface charge, however this effect was insignificant $(\mathrm{P}>0.05)$.

Basically, using a constant amount of TAC resulted in $\mathrm{EE} \%$ depending mainly on polymer concentration and DD. It was noticed in samples from A1 to B10 that increasing polymer concentration increased EE\% which was in accordance with Jones and Leroux ${ }^{29}$ who found that encapsulation efficiency (EE\%) depends on the aggregation number of the copolymer, where micelles showing greater aggregation number (number of monomers in a micelle) solubilized greater amounts of drug in their inner core. However, further increase in polymer concentration as in samples $\mathbf{A 2 0}$ and B20 showed no effect on EE\% as almost all drug molecules were encapsulated, and maximum loading capacities were reached (Table 3). When DD values increased, EE\% values increased as well which might be an outcome of increased hydrophobic regions within polymer backbone offering more hydrophobic interaction sites with TAC thereby encapsulating higher amount of the drug. ${ }^{30}$ From previous results, A10 and B10 were selected for further characterizations as they offered adequate particle size for passive targeting of the rheumatic tissue via extravasation (PS cut off $\sim 200$ $800 \mathrm{~nm}$ ) without being filtered by spleen (PS over $200 \mathrm{~nm}$ ) or eliminated by kidney (PS less than $10 \mathrm{~nm}$ ) besides having high zeta potential and the highest TAC EE\%. ${ }^{31}$

\section{Morphology of the Nano-Micelles}

TEM micrographs of the nano-micelles as shown in Figure 1C revealed spherical shapes for both A10 and B10. Their diameters ranged from $36 \mathrm{~nm}$ to $75 \mathrm{~nm}$, resembling those obtained by DLS technique (Figure 1D) with no obvious aggregations indicating stability of the prepared nano-micelles due to high surface charge. Apparent core-shell structure of polymeric micelles was noticed without any fragments or precipitates reflecting efficient drug encapsulation within the micellar core.

\section{DSC Thermographs and FTIR Spectra of Polymeric Nano-Micelles}

DSC thermograms of the nano-micelles A10, B10 as well as free TAC are shown in Figure 2A. Characteristic endothermic peak of free TAC was detected at $128^{\circ} \mathrm{C}$, which disappeared and overlapped from thermograms of nano-micelles samples A10 and B10; indicating occurrence of amorphization and entrapment of TAC within the core of polymeric nano-micelles.

The FT-IR spectra of MD- $\alpha$-TOC polymers, TAC@ MD- $\alpha$-TOC nano-micelles (A10 and B10) and free-TAC are shown in Figure 2B. Compatibility between the drug and tailored polymer was evidenced by absence of any chemical interactions. MD- $\alpha$-TOC bio-conjugates showed typical characteristic peaks of maltodextrin at $\mathrm{v} 3396$, 1141,1023 and $576 \mathrm{~cm}^{-1}$ which were ascribed to $\mathrm{O}-\mathrm{H}$ bond stretching; $\mathrm{C}-\mathrm{O}$ bending, $\mathrm{C}-\mathrm{O}-\mathrm{C}$ band stretching and anhydro-glucose ring stretching vibrations respectively. In addition, peaks of vitamin $\mathrm{E}$ appeared at $\mathrm{V}$ 1435 and $1390 \mathrm{~cm}^{-1}$ for $-\mathrm{CH} 3$ asymmetric/symmetric peaks, at $v 1226 \mathrm{~cm}^{-1}$ of $\mathrm{C}-\mathrm{O}$ stretching of phenol and $2924 \mathrm{~cm}^{-1}$ of C-H stretching along with ester bond specific peak at $v 1742 \mathrm{~cm}^{-1} \cdot{ }^{12}$ Moreover, TAC which has an empirical formula of $\mathrm{C}_{44} \mathrm{H}_{69} \mathrm{NO}_{12}$ showed characteristic peaks at $v 3457,1727,1639,1184$ and $1098 \mathrm{~cm}^{-1}$, corresponding to $\mathrm{O}-\mathrm{H}$ stretching vibration, $\mathrm{C}=\mathrm{O}$ (ester and ketone) stretching vibrations, $\mathrm{C}=\mathrm{O}$ (amide) and $\mathrm{C}=\mathrm{C}$ 

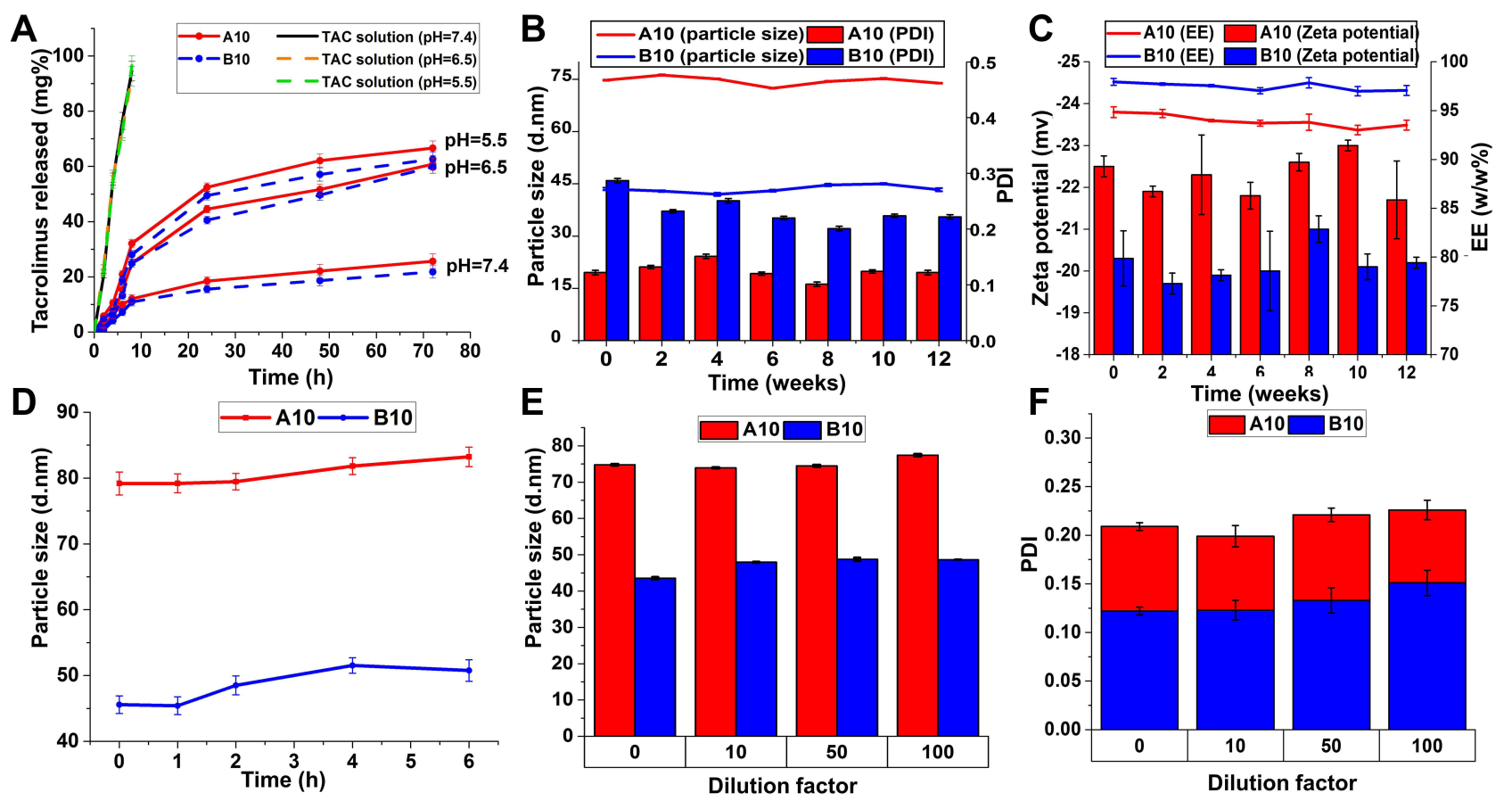

Figure 3 (A) In vitro drug release of TAC from AI 0, B I 0 and its ethanolic solution in PBS ( $\mathrm{pH} 7.5, \mathrm{pH} 6.5$ and $\mathrm{pH} 5.5)$ at $100 \mathrm{rpm}$ and $37^{\circ} \mathrm{C}$ using the dialysis bag method. Physical stability of AIO and BIO micelles stored at $4^{\circ} \mathrm{C}$ over 3 months expressed as (B) change in particle size, PDI, (C) zeta potential and EE\% with time. (D) Serum stability of AIO and B IO in 10\% fetal bovine serum (FBS) solution. Physical stability of AIO and B IO micelles at dilution factors (I0, 50, and I00) expressed as change in (E) particle size and (F) PDI.

stretching vibration, $\mathrm{C}-\mathrm{O}$ (ester) stretching vibration, and $\mathrm{C}-\mathrm{O}-\mathrm{C}$ (ether) stretching vibrations respectively. ${ }^{32}$

Overall, FTIR spectra of TAC@MD- $\alpha$-TOC micelles revealed that all the characteristic peaks of TAC and the polymer were in their original places without addition or deletion, indicating absence of strong chemical interaction with no incompatibility among all the formulation ingredients.

\section{$\mathrm{pH}$-Dependent Drug Release Profile of TAC from MD- $\alpha$-TOC Nano-Micelles}

Release patterns of TAC from A10 and B10 at different $\mathrm{pH}$ levels were studied (Figure 3A). Release profiles from the nano-micelles exhibited a biphasic pattern characterized by a burst initial release, followed by a pronounced slower and continuous release. At $\mathrm{pH}=7.4$, TAC release from A10 and B10 displayed an initial burst effect of 12\% and $11 \%$ within $8 \mathrm{~h}$ respectively. However, TAC release at $\mathrm{pH}=5.5$ and $\mathrm{pH}=6.5$ from $\mathbf{A 1 0}$ and $\mathbf{B 1 0}$ showed a faster release with an initial burst effect of $35 \%$ and $27 \%$ at $\mathrm{pH}=$ 5.5 and $28 \%$ and $25 \%$ at $\mathrm{pH}=6.5$, respectively within $8 \mathrm{~h}$. After $72 \mathrm{~h}$, the cumulative TAC released from A10 and B10 were 26 and $22 \%$, at $\mathrm{pH} 7.4$; while at $\mathrm{pH} 5.5$ and 6.5 they were $66 \%, 60 \%, 62 \%$ and $59 \%$, respectively. The initial burst release pattern could be attributed to the amount of TAC near the particle surface, while the slow and sustained released TAC arose from the diffusion of TAC from the micellar core. ${ }^{33}$ The marked faster release of TAC at acidic $\mathrm{pH}$ levels compared with that at $\mathrm{pH}=7.4$; this shows that TAC release from the MD- $\alpha$-TOC micelles is $\mathrm{pH}$ dependent. These results were in accordance with those obtained by Zhang et al. ${ }^{33}$ who reported an increased release rate of paclitaxel from Pluronic polymeric micelles upon reductions of $\mathrm{pH}$ value of the release medium. The enhanced release at $\mathrm{pH} 5.5$ and 6.5 could be ascribed to formation of hydrogen bonding between $\mathrm{H}+$ (predominant in the acidic media) and $\mathrm{OH}$ - groups of maltodextrin (outer shell of TAC@MD- $\alpha$-TOC nano-micelles) leading to the formation of more hydrophilic passages or channels that facilitated the diffusion of drug from the core. Furthermore, the relatively high negative zeta potential values of the nano-micellar formulations would enhance particle stability, preventing aggregation and thereby maintaining particles at their small particle size with large surface area in contact with release media for a longer period thus enhancing TAC release. On the other hand, released TAC from its ethanolic solution showed burst release reaching 77\% within $6 \mathrm{~h}$ without being affected by the $\mathrm{pH}$ values of the release media. ${ }^{34}$ 


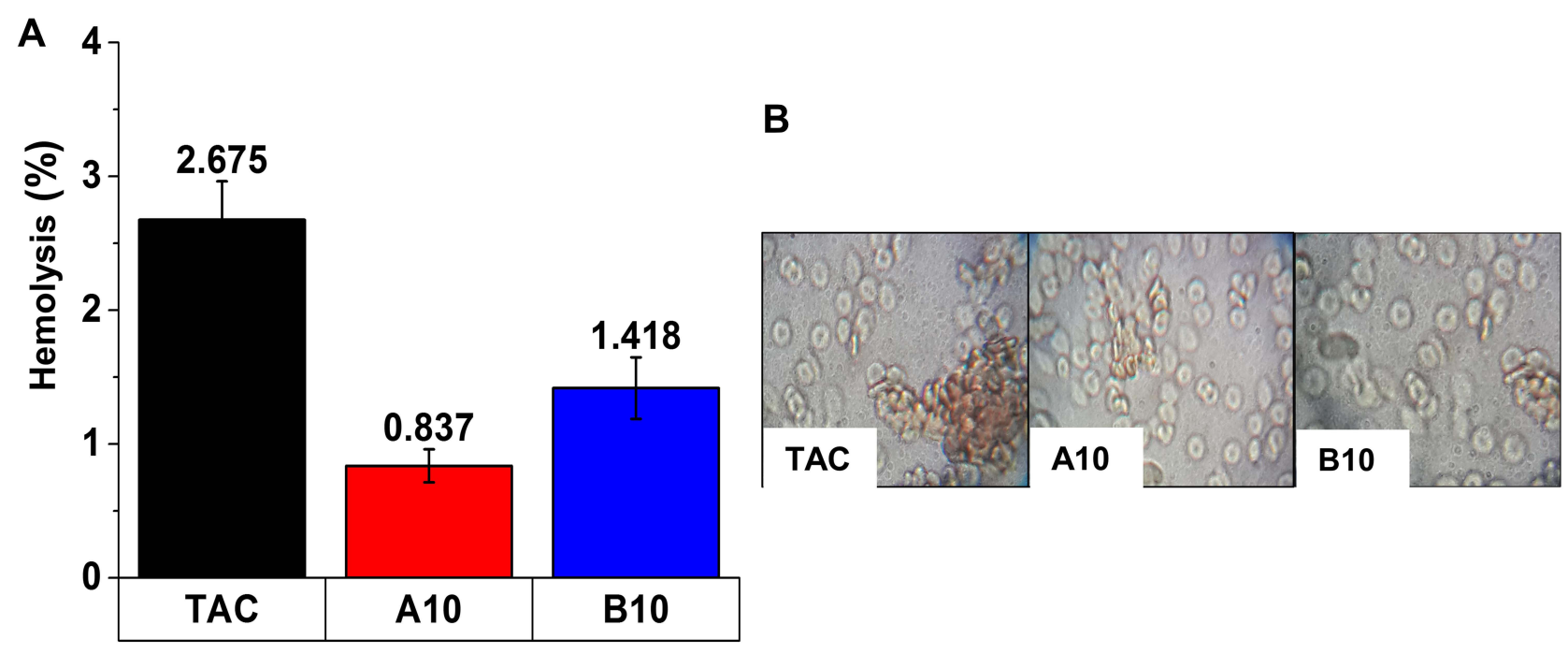

C
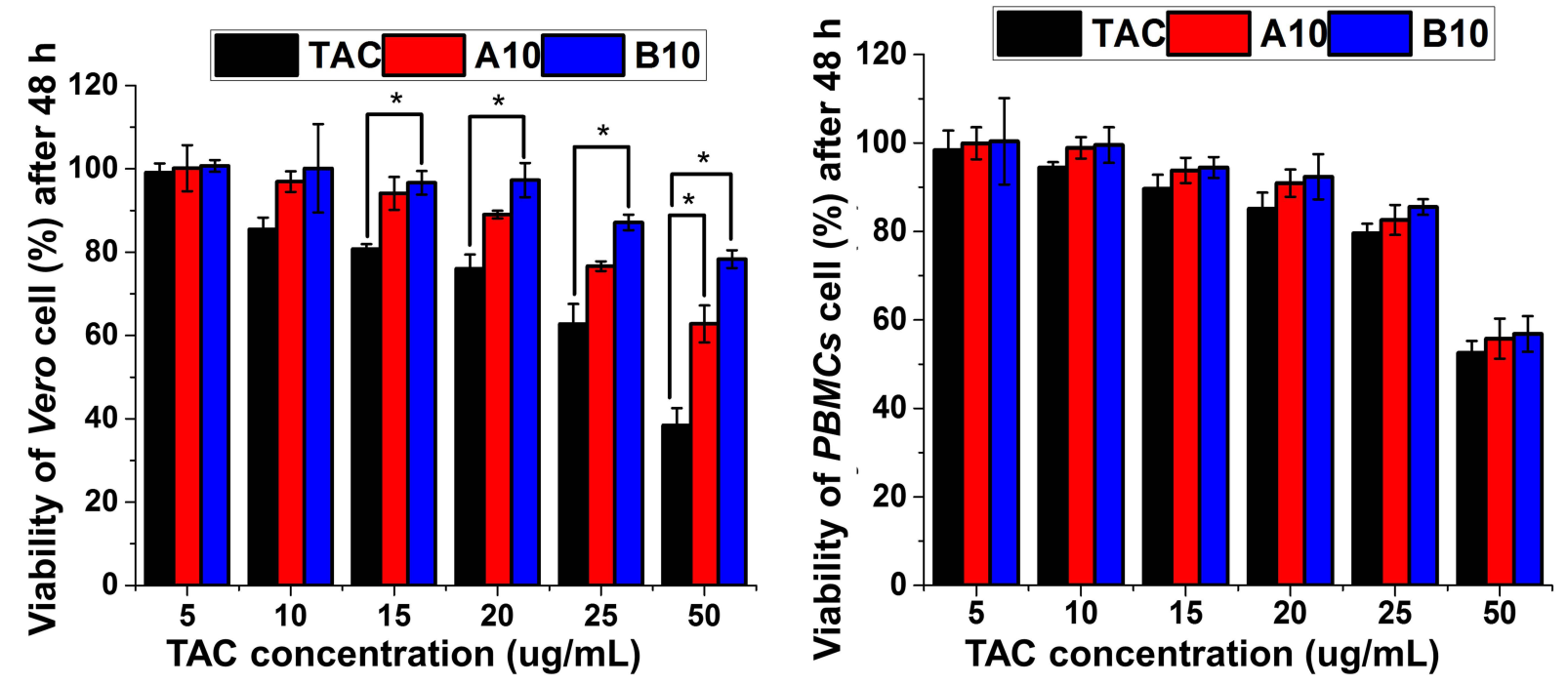

Figure 4 In vitro toxicological assessment (I): Hemolytic activity test of A 10 and B 10 expressed as (A) \% hemolysis (mean \pm SD) and (B) optical microscopic images of RBCs at the end of hemolysis test. Cytotoxicity test by MTT assay of prepared AIO and B 10 on (C) Vero kidney cell line and on (D) PBMCs cell line after $48 \mathrm{~h}$ of incubation [* P 0.05 vs $\mathrm{TAC}$, mean $\pm \mathrm{SD}]$.

The $\mathrm{pH}$-dependency of TAC release from the prepared bioconjugate micelles offered an extra merit to those tailored micellar structures as parenteral drug carriers aimed for rheumatoid arthritis. During the arthritis condition, the joint tissues are characterized by a decrease in physiological $\mathrm{pH}$ $(\mathrm{pH}=5-6.5),{ }^{35,36}$ thus the slow release of TAC from the bioconjugate nano-micelles at $\mathrm{pH}=7.4$ would improve the biostability of micelles at physiological conditions and preferentially release TAC at the site of inflamed joints.

The effect of degree of derivatization (DD\%) on the in vitro drug release from micellar structures was studied by comparing the release patterns from A10 and B10. B10 (with high DD) demonstrated a relatively lower release rate compared with A10 (with low DD), however the difference was not significant $(\mathrm{P}>0.05)$. This result was consistent with that obtained by Wei et al. ${ }^{30}$ who prepared hyaluronic aciddeoxycholic acid nano-micelles with different DD values and ascribed the reason of the influence of DD on in vitro drug release to the increased hydrophobic moieties within polymer backbone (core forming moiety) upon increasing DD; enhancing hydrophobic interaction between hydrophobic drug and hydrophobic core of nano-micelles. 


\section{In vitro Stability of Prepared MD- $\alpha-$ TOC Nano-Micelles}

To evaluate the stability of TAC@MD- $\alpha$-TOC nanomicelles, various parameters were taken into consideration including storage stability, interaction with serum and dilution with distilled water.

TAC loaded-nano-micelles were intended for longterm usage in the management of RA. Thus, maintenance of their physicochemical properties during storage period was a mandatory determination. There was no significant change $(\mathrm{P}>0.05)$ in particle size, PDI and zeta potential values during the entire storage periods (Figure $3 \mathrm{~B}$ and $\mathrm{C}$ ). A10 and B10 nano-micelles showed particle size of 73.92 \pm 1.11 and $43.31 \pm 0.43 \mathrm{~nm}$ respectively after 3 months of storage, compared with the initially stored $(74.78 \pm 1.14$ and $43.53 \pm 0.33$ ) micelles. Moreover, content of TAC within the micellar structures did not change significantly and no changes in color nor precipitation was observed for the micellar dispersions during the whole storage period.

TAC is a highly lipophilic drug which is highly bound to blood and plasma components with an unbound or pharmacologically active fraction of $\sim 1-2 \%{ }^{37}$ Also, since the prepared micelles were intended for parenteral drug delivery, adsorption of serum proteins onto the nano-micelles was considered one of the major challenges. Figure 3D demonstrated insignificant change in particle size $(\mathrm{P}<0.05)$ during the whole incubation period $(6 \mathrm{~h})$. This together with absence of any aggregates formation during the incubation provided evidence of absence of serum proteins adsorption on the surface of nano-micelles samples. These results supported the selection of tailored bioconjugate nano-micelles as carriers for TAC to prevent interaction of TAC with serum proteins. Thus, increasing the abundance of unbound drug portion that would subsequently decrease the initial effective dose required of TAC, which in turn would help in decreasing its substantial side effects.

Another challenge facing IV administered polymeric nano-micelles is the dilution with physiological fluids that might lead to nano-micelles instability. DLS data displayed in Figure $3 \mathrm{E}$ and $\mathrm{F}$ revealed that upon dilution of the nano-micellar dispersion, no significant change in particle size or PDI $(\mathrm{P}>0.05)$ occurred, indicating absence of dissociated micelles at different dilution ratios. These promising stability results could probably be ascribed to the apparently low $\mathrm{CMC}$ of the synthesized bioconjugates leading to resistance of formed micelles to dissociate. This gives an indication that upon in vivo administration and dilution with physiological fluids, micellar structures integrity of the biopolymer will be maintained, thus overcoming the instability problems encountered with conventional micelles from detergent-like polymers. ${ }^{12,17}$

\section{In vitro and in vivo Toxicological Assessment}

TAC has serious toxicological dose-dependent hazards such as hyperglycemia, hyperkalemia, vaso-constrictive properties, decrease in neutrophil counts and more seriously nephrotoxicity. ${ }^{38}$ Thus, preventing TAC accompanied side effects without decreasing its dose to obtain the desired therapeutic outcomes is one of the basic aims for utilizing MD- $\alpha$-TOC based polymeric micelles as TAC nanocarriers.

\section{Hemolytic Activity Assay}

According to the American Society for Testing and Materials (ASTM E2524), nanoparticles' activities on RBCs could be classified into three different categories: hemolytic (hemolysis $\geq 5 \%$ ), slightly hemolytic (between $2 \%$ and $5 \%)$, and non-hemolytic $(\leq 2 \%){ }^{39}$ TAC is known to be mainly distributed in red blood cells (RBCs) after IV injections. ${ }^{40}$ It showed a slight hemolytic activity on RBCs with hemolysis $\% \sim 2.675 \%$. Blank nano-micelles were previously reported to have a high safety profile on the integrity of RBCs. ${ }^{12}$ In vitro interaction of TAC@MD- $\alpha$ TOC nano-micellar structures with RBCs demonstrated the absence of any hemolytic activity of the nanomicellar dispersions as their \% hemolysis were $0.837 \%$ and $1.418 \%$ for A10 and B10, respectively (Figure 4A and B), indicating safety of the micellar dispersions upon IV injections. These results could be attributed to the negative charge on the surface of nanoparticles (due to maltodextrin domains), which decreased interaction with RBCs due to repulsive forces. ${ }^{16}$

\section{In vitro Cytotoxicity Test by MTT Assay}

As an emphasis on the safety and privilege of TAC@MD- $\alpha$-TOC nano-micelles in decreasing TAC accompanied nephrotoxicity, MTT assay on Vero kidney cells was performed (Figure 4C). Our findings revealed that TAC@MD- $\alpha$-TOC nano-micelles reserved the viability of the Vero cells compared with free TAC. A pronounced decrease in cell viability was observed after $48 \mathrm{~h}$ incubation with free TAC at $50 \mu \mathrm{g} / \mathrm{mL}$ with viability $\sim 39 \%$. On the other hand, the viability of Vero 

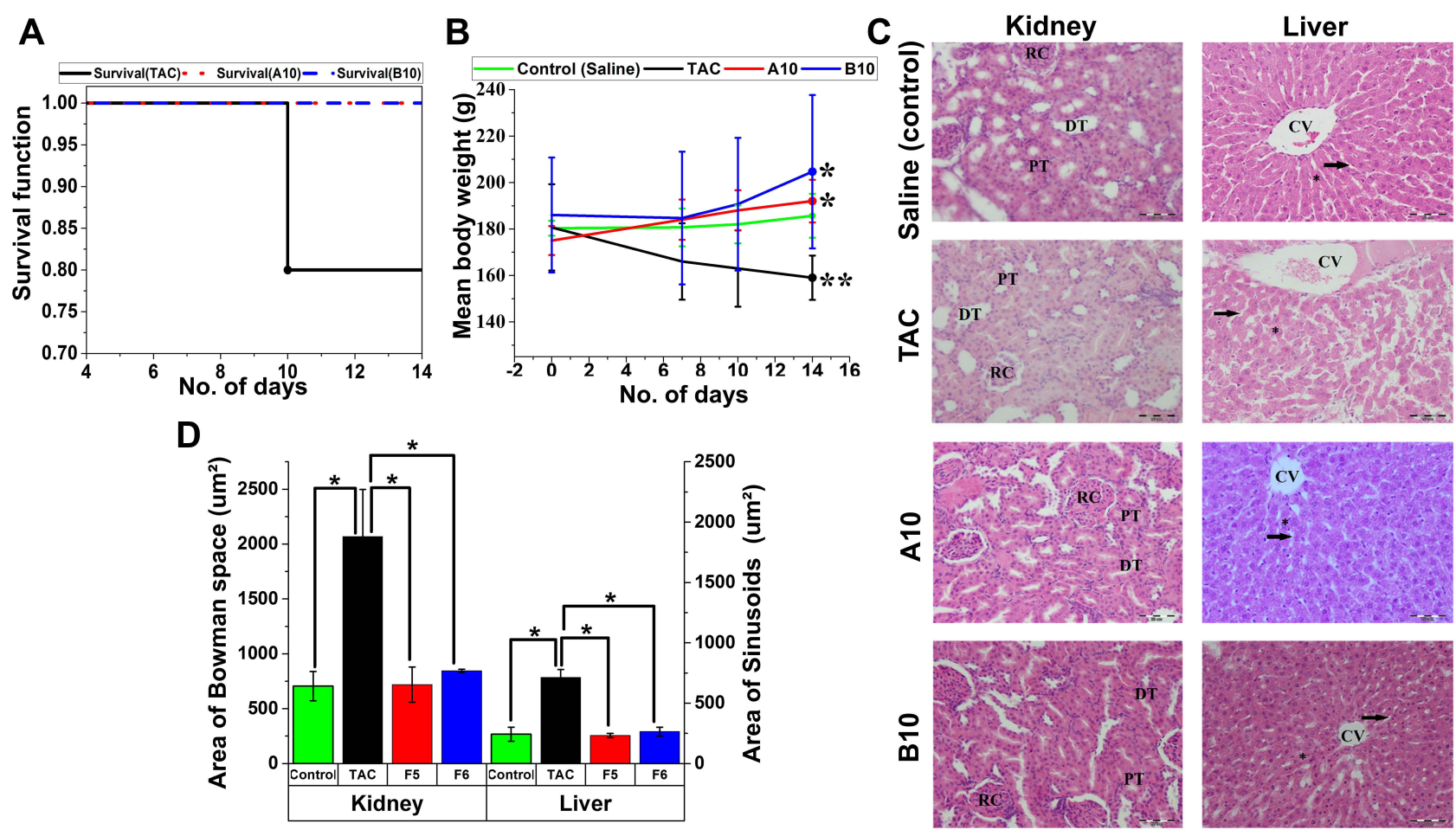

Figure 5 In vivo toxicological assessment (II): (A) Kaplan-Meier survival curve for the overall survival of rats during sub-acute toxicity study. (B) Mean body weight (mean \pm SEM) [* significantly increase vs 0 -time, ** significantly decrease vs 0 -time, $\mathrm{P} \leq 0.05$ ]. (C) Photomicrographs of $\mathrm{H}$ and $\mathrm{E}$ stained kidney sections of rats treated with TAC, Al0, and B 10 showing renal cortex of renal corpuscle (RC), proximal (PT) and distal (DT) tubules and liver sections showing central vein (CV), radiated hepatic cords (*) and hepatic sinusoids (arrows) (original magnifications $\times 400$ ) and (D) kidney (Bowman space area) and liver (sinusoids area) histological scores of TAC, Al0, and BI 0 treated groups [* $\mathrm{P} \leq 0.05$ vs TAC, mean $\pm \mathrm{SD}$ ].

cells did not reach values less than $63 \%$ and $78 \%$ after $48 \mathrm{~h}$ incubation with $\mathbf{A 1 0}$ and B10, respectively. These results provide evidence that the efficient encapsulation of TAC within the bioconjugate nano-micelles had the effect of decreasing its toxicity without dose reduction. It was also noticed that nano-micelles B10 maintained the Vero cells viability to greater extent, compared with A10. This effect could be attributed to the higher hydrophobic interaction between $\alpha$-TOC domains within B10 structure and TAC leading to slower drug release decreasing TAC toxicity.

PBMCs are the peripheral blood mononuclear cells representing human lymphocytes, monocytes and dendritic cells that are functionally evaluated by proliferation, survival, and cytotoxicity analysis. ${ }^{41}$ MTT assays on PBMCs demonstrated no significant impact on the cells' viability after $48 \mathrm{~h}$ incubation for all test samples up to concentration of $25 \mu \mathrm{g} / \mathrm{mL}$ giving an indication on the lack of toxicity for both TAC and its loaded micelles on blood lymphocytes upon IV injection (Figure 4D).

\section{In vivo Sub-Acute Toxicity Study}

TAC was reported to induce renal glomerular injury by formation of reactive oxygen species (ROS) and disturbance in antioxidant defense. It was also reported to induce hepatotoxicity by increasing the intrahepatocellular ROS and inhibition of nitric oxide. ${ }^{42}$ The sub-acute toxicity study was performed to evaluate the safety of TAC@MD- $\alpha$-TOC nano-micelles compared with free TAC on the vital excretory organs (liver and kidney). The nano-micelles treated group showed 100\% survival rate as shown in Figure 5A. On the other hand, the free TAC treated group exhibited a $20 \%$ mortality rate. Rats that received the nano-micelles had normal motor activities during the whole study with increased body weight and diminished loss of appetite (Figure 5B), while rats that received free TAC suffered from apparent loss of appetite, concomitant diarrhea with decreased body weight, which might be owed to TAC side effects on the gastrointestinal tract (GIT). ${ }^{43}$

Hematological and biochemical parameters of all treated groups are shown in Tables 4 and 5. Free TAC had 

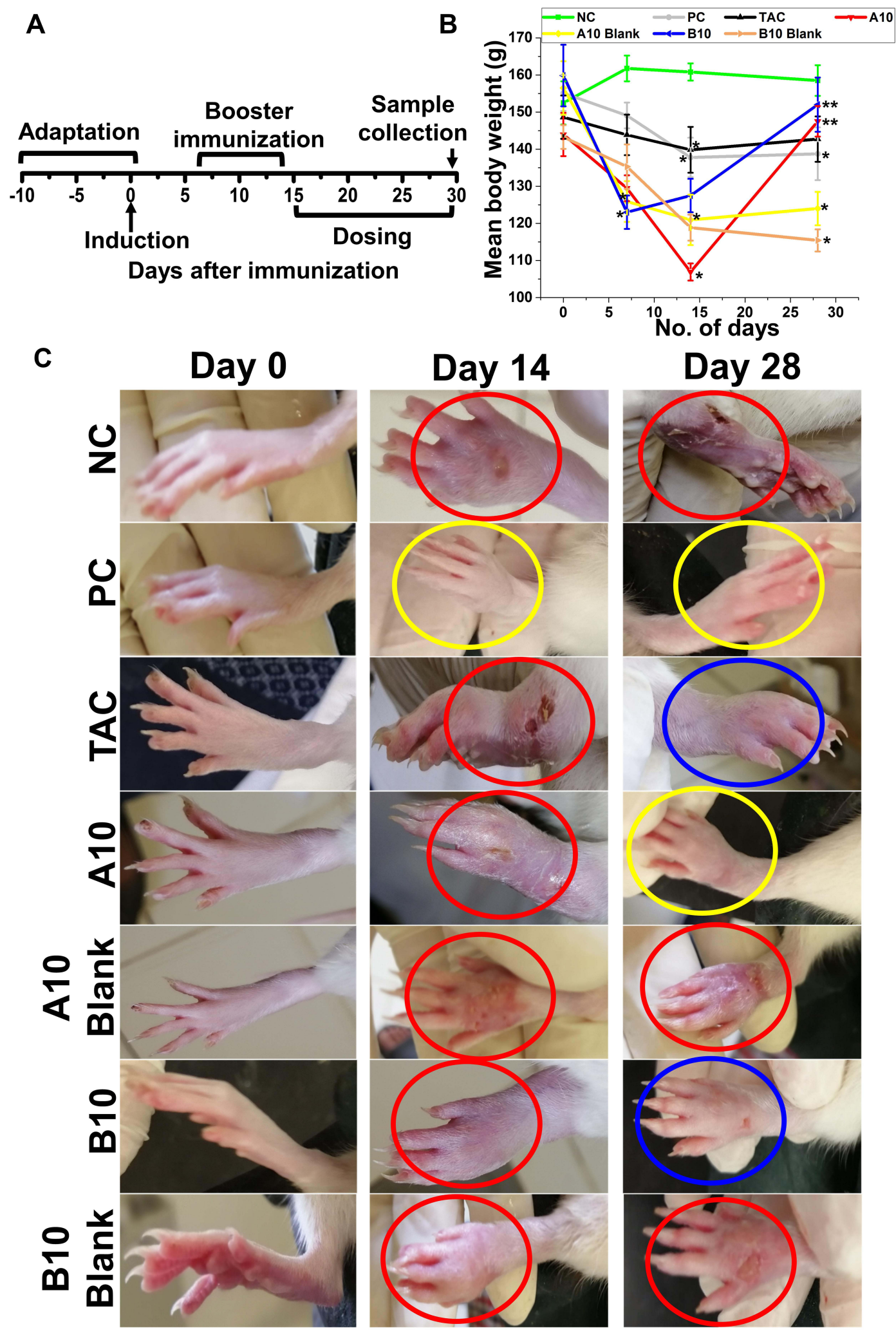

Figure 6 Assessment of AIA model (I): (A) Experimental timeline of the AIA model used showing induction and treatment protocols. (B) Mean body weights (mean \pm SEM) during induction and treatment period [** significantly increase vs 14 days reading, * significantly decrease vs 0 -time, $P \leq 0.05$ ]. (C) Images of hind paws of different treated groups at 0,14 - and 28 -days post-induction $(\mathrm{NC}=$ negative control and $\mathrm{PC}=$ positive control), $[$ red circle $=$ severe inflammation, blue circle $=$ mild inflammation, yellow circle= no inflammation]. 
Table 4 Effect of Acute IV Administration of Free TAC, A I 0, B IO on Hematological Parameters in Rats

\begin{tabular}{|c|c|c|c|c|c|c|c|c|}
\hline & \multicolumn{2}{|c|}{ Control (Saline) } & \multicolumn{6}{|c|}{ Treatment Groups } \\
\hline & \multirow{2}{*}{$\begin{array}{l}\text { Zero } \\
\text { Time }\end{array}$} & \multirow{2}{*}{$\begin{array}{c}\text { After I4 } \\
\text { Days }\end{array}$} & \multicolumn{2}{|c|}{ TAC } & \multicolumn{2}{|c|}{ AlO } & \multicolumn{2}{|c|}{ B IO } \\
\hline & & & $\begin{array}{l}\text { Zero } \\
\text { Time }\end{array}$ & $\begin{array}{l}\text { After I4 } \\
\text { Days }\end{array}$ & $\begin{array}{l}\text { Zero } \\
\text { Time }\end{array}$ & $\begin{array}{c}\text { After I4 } \\
\text { Days }\end{array}$ & $\begin{array}{l}\text { Zero } \\
\text { Time }\end{array}$ & $\begin{array}{c}\text { After I4 } \\
\text { Days }\end{array}$ \\
\hline & & & \multicolumn{3}{|c|}{ Hematological parameters } & & & \\
\hline HGB (mg/dL) & $14.08 \pm 0.5$ & $14.5 \pm 0.5$ & $14.1 \pm 0.1$ & $15.4 \pm 0.6$ & $12.9 \pm 0.4$ & $14.3 \pm 0.8$ & $15.5 \pm 0.8$ & $14.7 \pm 0.6$ \\
\hline HCT (\%) & $43.8 \pm 1.5$ & $47.9 \pm 1.6^{a}$ & $42.5 \pm 0.3$ & $50.7 \pm 1.4^{\mathrm{a}}$ & $47 \pm 1.9$ & $48.3 \pm 1.4$ & $47.2 \pm 0.9$ & $46.4 \pm 0.7$ \\
\hline $\operatorname{RBC}\left(\times 10^{6} \mu \mathrm{L}\right)$ & $8.64 \pm 0.05$ & $8.75 \pm 0.05$ & $7.64 \pm 0.2$ & $8.43 \pm 0.1$ & $7.58 \pm 0.4$ & $8.63 \pm 0.3$ & $9.18 \pm 0.1$ & $8.57 \pm 0.3$ \\
\hline WBC $\left(\times 10^{3} \mu \mathrm{L}\right)$ & $19.6 \pm 0.2$ & $19.7 \pm 0.2$ & $18.7 \pm 0.2$ & $19.1 \pm 0.6$ & $18.7 \pm 1.1$ & $21.6 \pm 1.5$ & $17.5 \pm 1.7$ & $18.7 \pm 1.6$ \\
\hline Neutrophils (\%) & $12.8 \pm 0.4$ & $12.5 \pm 0.5$ & $11.6 \pm 0.4$ & $10.7 \pm 0.6$ & $13.4 \pm 0.2$ & $13 \pm 0.4$ & $13.6 \pm 0.5$ & $14.2 \pm 0.3$ \\
\hline Lymphocytes (\%) & $82.3 \pm 0.8$ & $83.5 \pm 0.8$ & $82.3 \pm 1.2$ & $82.3 \pm 0.8$ & $82.5 \pm 0.9$ & $82 . I \pm 0.8$ & $80.3 \pm 1.2$ & $81.6 \pm 3.9$ \\
\hline Monocytes (\%) & $1.5 \pm 0.15$ & $1.2 \pm 0.21$ & $1.8 \pm 0.2$ & $1.6 \pm 0.2$ & $1.8 \pm 0.2$ & $1.6 \pm 0.2$ & $1.9 \pm 0.4$ & $1.9 \pm 0.4$ \\
\hline Eosinophils (\%) & $1.8 \pm 0.2$ & $1.3 \pm 0.1^{\mathrm{a}}$ & $2.2 \pm 0.1$ & $2.5 \pm 0.6$ & $2 \pm 0.07$ & $1.8 \pm 0.1$ & $1.8 \pm 0.1$ & $1.7 \pm 0.07$ \\
\hline Basophils (\%) & $1.8 \pm 0.2$ & $1.5 \pm 0.1$ & $2.8 \pm 0.4$ & $2.8 \pm 0.7$ & $1.8 \pm 0.2$ & $1.9 \pm 0.2$ & $1.7 \pm 0.1$ & $1.6 \pm 0.1$ \\
\hline RDW (\%) & $17.3 \pm 0.4$ & $17.9 \pm 0.3^{\mathrm{a}}$ & $13.6 \pm 0.7$ & $15.5 \pm 1.1$ & $16.4 \pm 0.4$ & $17 \pm 0.8$ & $18.2 \pm 0.9$ & $19.4 \pm 0.5$ \\
\hline $\mathrm{MCV}(\mathrm{fL})$ & $50.5 \pm 1.4$ & $54.7 \pm 1.4^{\mathrm{a}}$ & $56.4 \pm 0.5$ & $60.3 \pm 2.7$ & $56.7 \pm 3$ & $54.6 \pm 1.2$ & $51.6 \pm 2$ & $55.2 \pm 2.7$ \\
\hline $\mathrm{MCH}(\mathrm{pg})$ & $16.2 \pm 0.3$ & $16.5 \pm 0.2$ & $18.6 \pm 0.2$ & $|8| \pm 0.3$. & $17.5 \pm 0.4$ & $16.1 \pm 0.7^{a}$ & $16.8 \pm 0.3$ & $16.7 \pm 0.4$ \\
\hline $\mathrm{MCHC}(\mathrm{mg} / \mathrm{dL})$ & $32.1 \pm 1.5$ & $30.2 \pm 0.8$ & $33 \pm 0.5$ & $30.3 \pm 0.7^{\mathrm{a}}$ & $32.8 \pm 1.05$ & $29.4 \pm 1.1$ & $32.8 \pm 0.6$ & $30.9 \pm 1.1$ \\
\hline PLT $\left(\times 10^{3} \mu \mathrm{L}\right)$ & $1206 \pm 9.6$ & $1153 \pm 13.6^{a}$ & $969 \pm 7.8$ & $1055 \pm 37.3$ & $976 \pm 6.2$ & $1167 \pm 34.3$ & $1079 \pm 15.1$ & $1027 \pm 19.5$ \\
\hline
\end{tabular}

Notes: Values are given as mean $( \pm$ S.E.M. $n=5)$. ${ }^{a}$ : indicates significant difference with the same group at zero time.

Table 5 Effect of Acute IV Administration of Free TAC, A I 0, B I0 on Biochemical Parameters in Rats

\begin{tabular}{|c|c|c|c|c|c|c|c|c|}
\hline & \multicolumn{2}{|c|}{ Control (Saline) } & \multicolumn{6}{|c|}{ Treatment Groups } \\
\hline & \multirow{2}{*}{$\begin{array}{l}\text { Zero } \\
\text { Time }\end{array}$} & \multirow{2}{*}{$\begin{array}{c}\text { After I4 } \\
\text { Days }\end{array}$} & \multicolumn{2}{|c|}{ TAC } & \multicolumn{2}{|c|}{ Alo } & \multicolumn{2}{|c|}{ B 10} \\
\hline & & & $\begin{array}{l}\text { Zero } \\
\text { Time }\end{array}$ & $\begin{array}{c}\text { After I4 } \\
\text { Days }\end{array}$ & $\begin{array}{l}\text { Zero } \\
\text { Time }\end{array}$ & $\begin{array}{l}\text { After I4 } \\
\text { Days }\end{array}$ & $\begin{array}{l}\text { Zero } \\
\text { Time }\end{array}$ & $\begin{array}{c}\text { After I4 } \\
\text { Days }\end{array}$ \\
\hline \multicolumn{9}{|c|}{ Biochemical parameters } \\
\hline Urea (mg/dL) & $47.3 \pm 2.4$ & $46.3 \pm 2.2$ & $44.1 \pm 1.9$ & $48.3 \pm 1.9^{\mathrm{a}}$ & $38.7 \pm 1.6$ & $40.9 \pm 2.1$ & $43.8 \pm 3.1$ & $40.5 \pm 2.1$ \\
\hline Creatinine $(\mathrm{mg} / \mathrm{dL})$ & $0.49 \pm 0.02$ & $0.5 \mathrm{I} \pm 0.02$ & $0.5 I \pm 0.06$ & $0.94 \pm 0.1^{\mathrm{a}}$ & $0.46 \pm 0.04$ & $0.48 \pm 0.03$ & $0.44 \pm 0.03$ & $0.43 \pm 0.05$ \\
\hline AST (IU/L) & $97.5 \pm 3.8$ & $11 I \pm 5.9$ & $106 \pm 9.5$ & $769 \pm 39^{a}$ & $156 \pm 19.4$ & $124 \pm 20.7$ & $|44 \pm| 5 . \mid$ & $|45 \pm| 4.4$ \\
\hline ALT (IU/L) & $67.1 \pm 2.4$ & $66.9 \pm 2.7$ & $68.5 \pm 2.1$ & $72.3 \pm 3.9$ & $80.5 \pm 3.5$ & $82.3 \pm 3.6$ & $88.2 \pm 5.5$ & $84.5 \pm 3.7$ \\
\hline
\end{tabular}

Notes: Values are given as mean $( \pm$ S.E.M. $n=5) .{ }^{a}$ indicates significant difference with the same group at zero time.

a negligible effect on hematological parameters of treated rats. This result was similar to that obtained by Akar et al. ${ }^{38}$ who reported that IV-administered TAC at a dose of $1 \mathrm{mg} / \mathrm{kg}$ had a negligible effect on hematological parameters of treated groups. On the other hand, parameters measuring renal functions (serum urea nitrogen concentration and creatinine) showed significantly $(\mathrm{P}<0.05)$ elevated levels with free TAC administration in comparison with the control group. Moreover, the administration of TAC@MD- $\alpha$-TOC nanomicelles showed normal values for hematological parameters, renal and hepatic functions indicating decrease in TAC nephrotoxicity without affecting normal biochemical parameters. Reasons behind the obtained results could be the efficient TAC encapsulation in nano-micellar core, slow rate of TAC release from nano-micellar structures along with selection of two natural substrates (maltodextrin and $\alpha$-tocopherol) in the preparation of the amphiphilic polymer reducing excipients' side effects and enhancing antioxidant activity (see Figure S4, Supporting Information). $^{12}$

Histopathological examination of liver and kidney tissues is illustrated in Figure 5C. Samples from rats that received free TAC indicated prominent renal toxicological signs such as tubular necrosis, edema of interstitial spaces, attenuated epithelium of proximal tubules with loss of brush borders along with dilated tubules and Bowman spaces in 


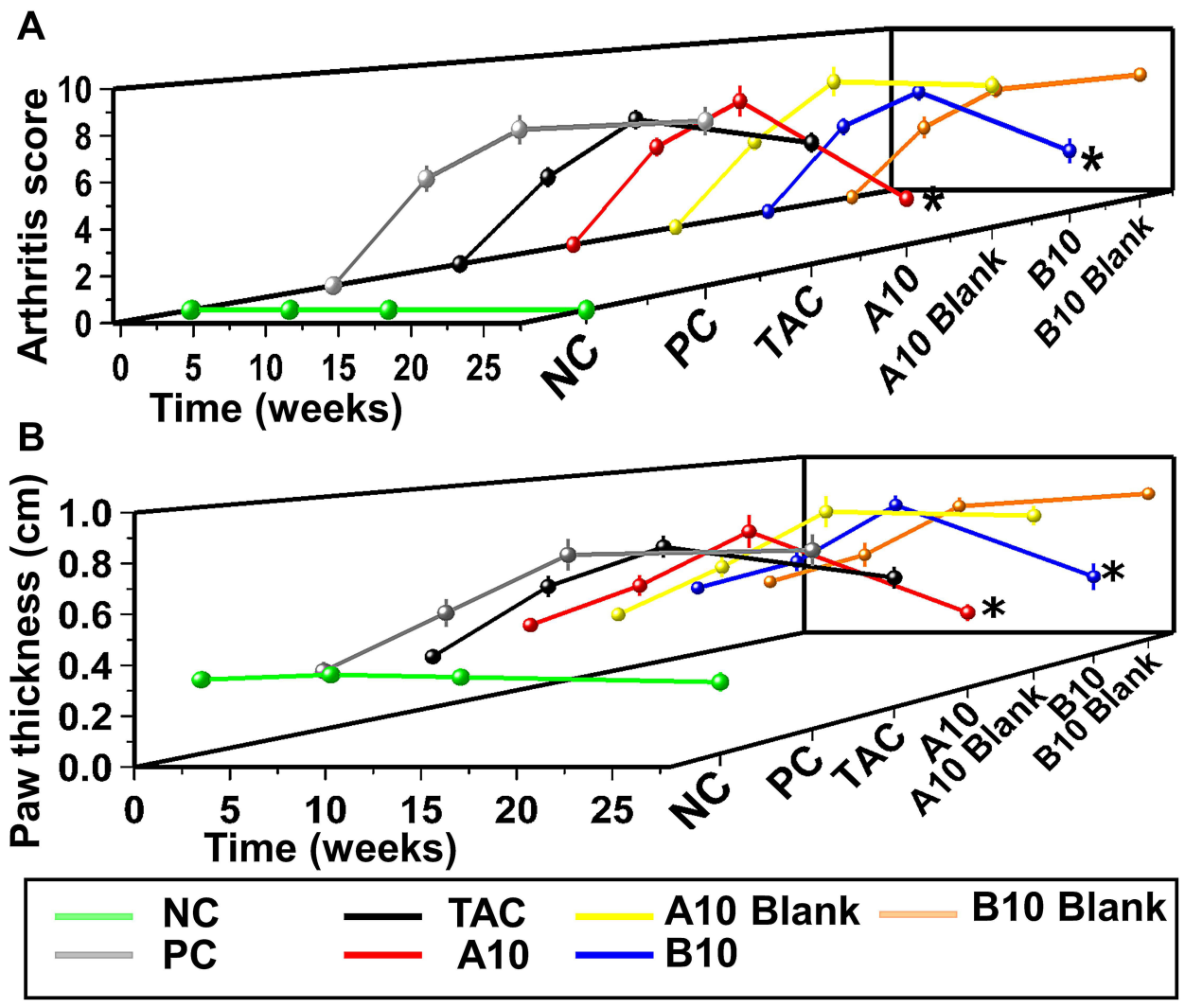

Figure 7 Assessment of AIA model (II): (A) Clinical arthritis score (mean \pm SEM) [* significantly decreased after treatment, $P \leq 0.05]$ and (B) paw thickness (mean \pm SEM) [* significantly decreased after treatment, $P \leq 0.05]$.

Abbreviations: NC, negative control; PC, positive control.

comparison with control group (received saline). On the other hand, renal toxicological signs were significantly diminished $(\mathrm{P}<0.05)$ (Figure 5D) with nano-micelles treated groups showing almost normal tubules and glomerulus.

The particle size, zeta potential along with the nature of tailored polymer utilized in TAC nano-micelles had the upper hand in decreasing its nephrotoxicity. Both A10 and B10 were prepared to have particle sizes ranging from $30-80 \mathrm{~nm}$ which were far from sizes prone to distribution in the kidney (less than $10 \mathrm{~nm}$ ). ${ }^{44,45}$ Furthermore, it was previously reported that negatively charged nanocarriers had slow glomerular filtration. ${ }^{45}$ Also, the tailored MD- $\alpha$-TOC conjugates possess enhanced antioxidant activities, compared with native vitamin E succinate (see Figure S4, Supporting Information) which had a mark in preserving kidney's architecture and preventing nephrotoxicity associated with free TAC. ${ }^{12,46}$

Liver histology of the free TAC group revealed disturbed liver architecture with apparent widening of sinusoids, congestion of central vein and nuclear changes in comparison with control. Treatment with loaded nanomicelles had a negligible effect on liver structure with regular arrangement of hepatocytes and apparent radiating hepatic cords (Figure 5C). Additionally, TAC@MD- $\alpha$ TOC micelles significantly $(\mathrm{P}<0.05)$ decreased hepatic toxicological signs accompanied with TAC (Figure 5D).

\section{Adjuvant-Induced Arthritis (AIA) Model in Rats \\ Assessment of AIA Model}

Induction of rheumatoid arthritis in female Wistar rats was confirmed on the 7th day with the start of the inflammation and paw edema. On the 14th day, and after the third dose of CFA, all rats developed a maximum swollen paw and ankle. Induction and treatment protocols are illustrated in Figure 6A. After induction of the AIA model, all rats experienced a significantly $(\mathrm{P}<0.05)$ decreased body weight due to pain and inflammation leading to loss of appetite. However, body weights of rats in groups receiving $\mathbf{A 1 0}$ and B10 showed a significant increase at the end of treatment period. On the other hand, body weights of other treated groups demonstrated non-significant $(\mathrm{P}>0.05)$ increase at the 
A
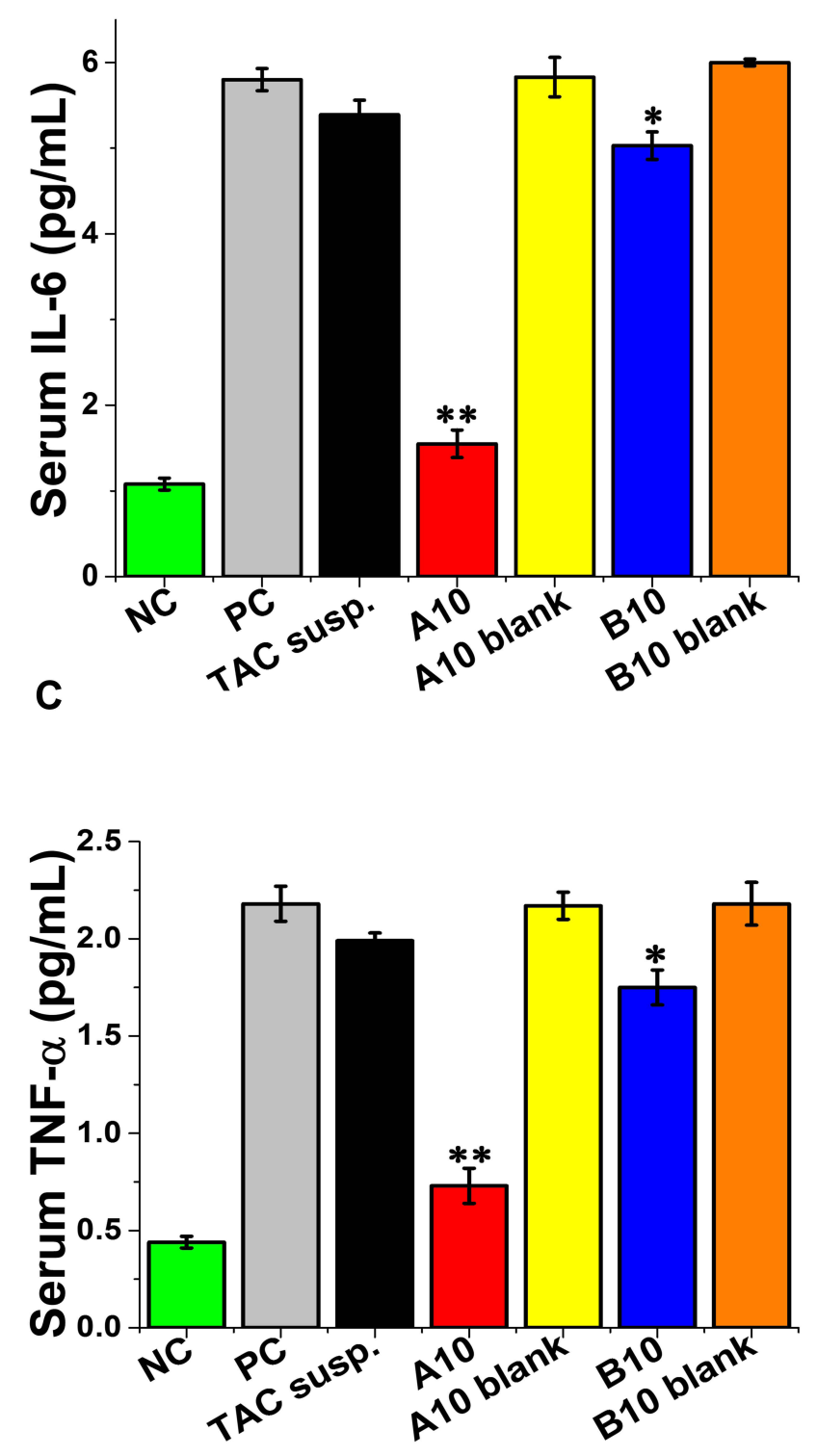

B
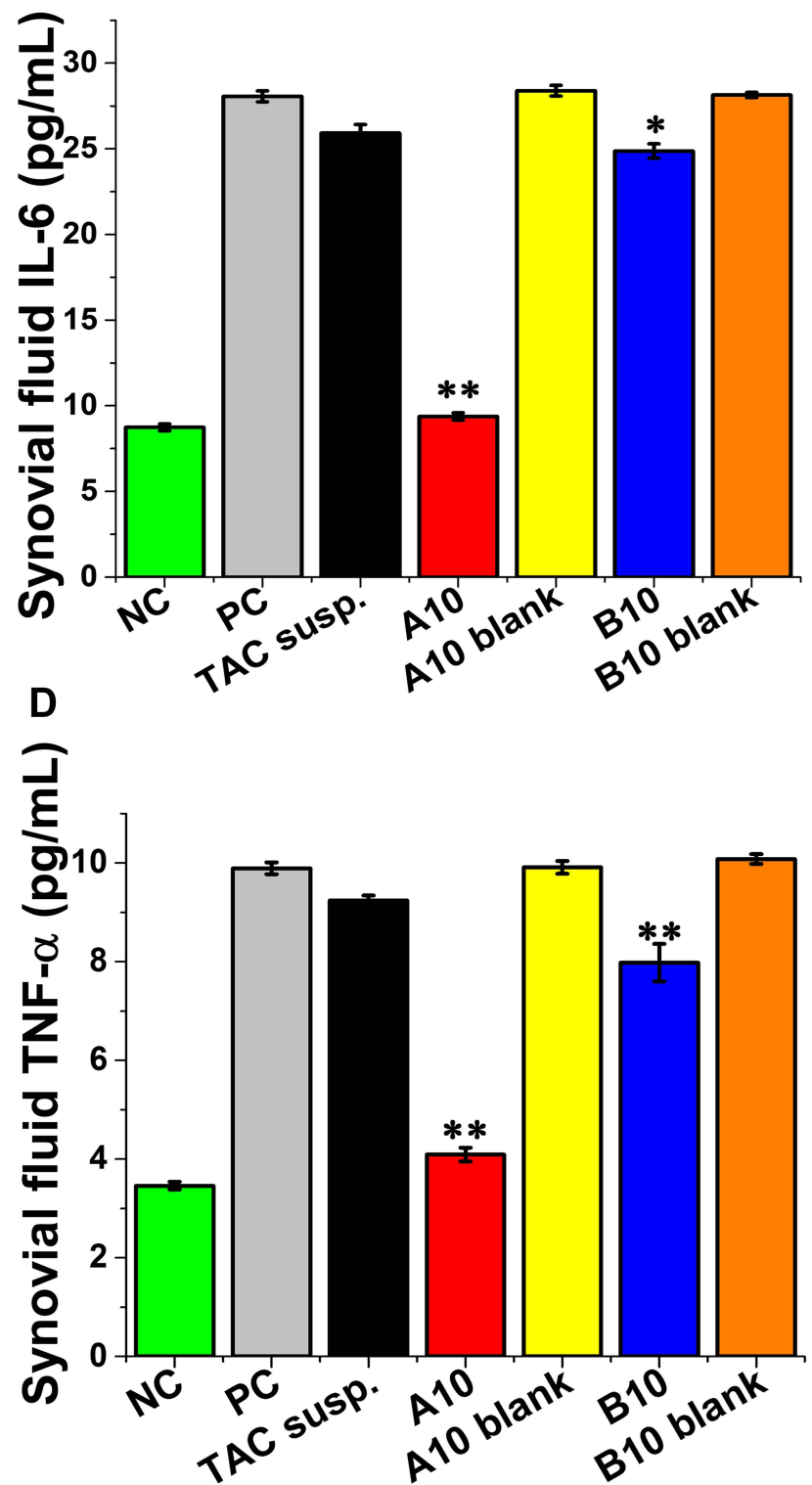

Figure 8 Concentration of pro-inflammatory cytokines. (A) Serum level of IL-6, (B) synovial fluid level of IL-6, (C) serum level of TNF-a, and (D) synovial fluid level of TNFa $[* \mathrm{P} \leq 0.05$ vs PC, ** P $\leq 0.05$ vs PC and TAC groups, mean \pm SEM, $n=5]$.

Abbreviations: NC, negative control; PC, positive control.

end of treatment (Figure 6B). During the whole study, the mortality rate of all groups was $0 \%$.

Clinical signs of paws and ankles are shown in Figure 6C. An increase in frequency and severity in hind paws erythema and swelling was noticed with all groups before starting the treatment regimen. However, at the end of the treatment cycle all the treated groups but not the control ones experienced a reduction in the frequency and severity of clinical arthritis scores to different extents, where maximum reduction was observed with A10 (Arthritis score $=2.4 \pm 0.416$ ) followed by B10 then free TAC. Groups which received blank nano-micelles did not show significant decrease in the inflammation of paws and ankles (Figure 7A).

Paw thickness was evidence of the inflammatory reaction accompanied with induction of rheumatoid arthritis. Figure 7B illustrated the influence of different treatment regimens on the paws thickness. The paw thickness of different groups increased significantly until reaching its maximum value on the 14th day (maximum paw thickness $=0.8 \pm 0.082 \mathrm{~cm}$ ). At the end of treatment, paw thickness significantly decreased 

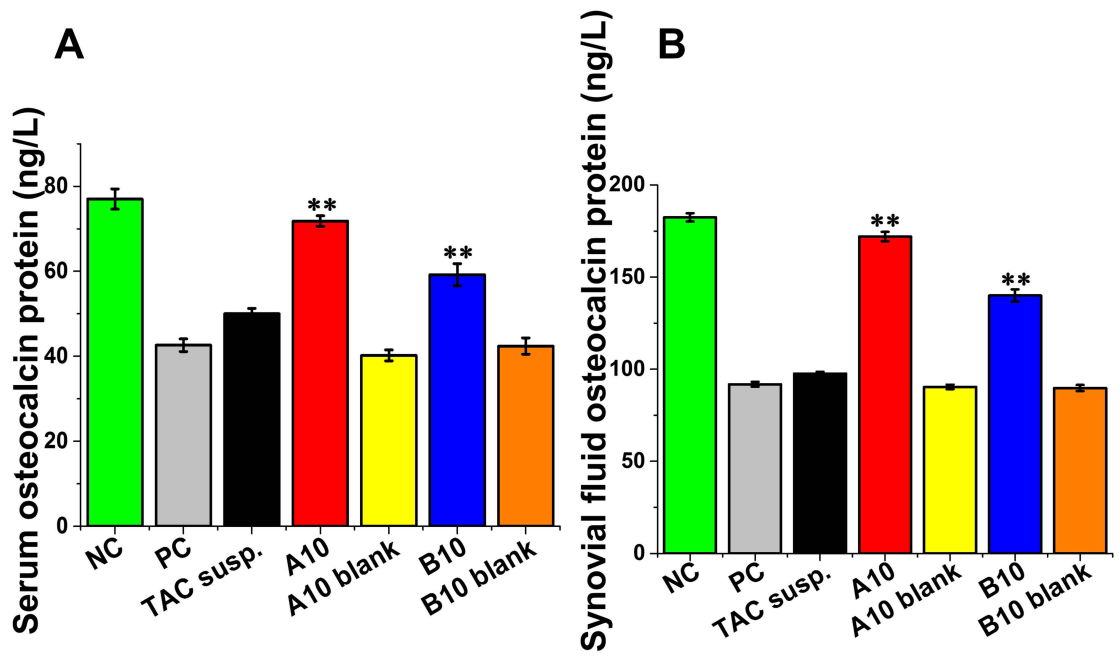

\section{C}
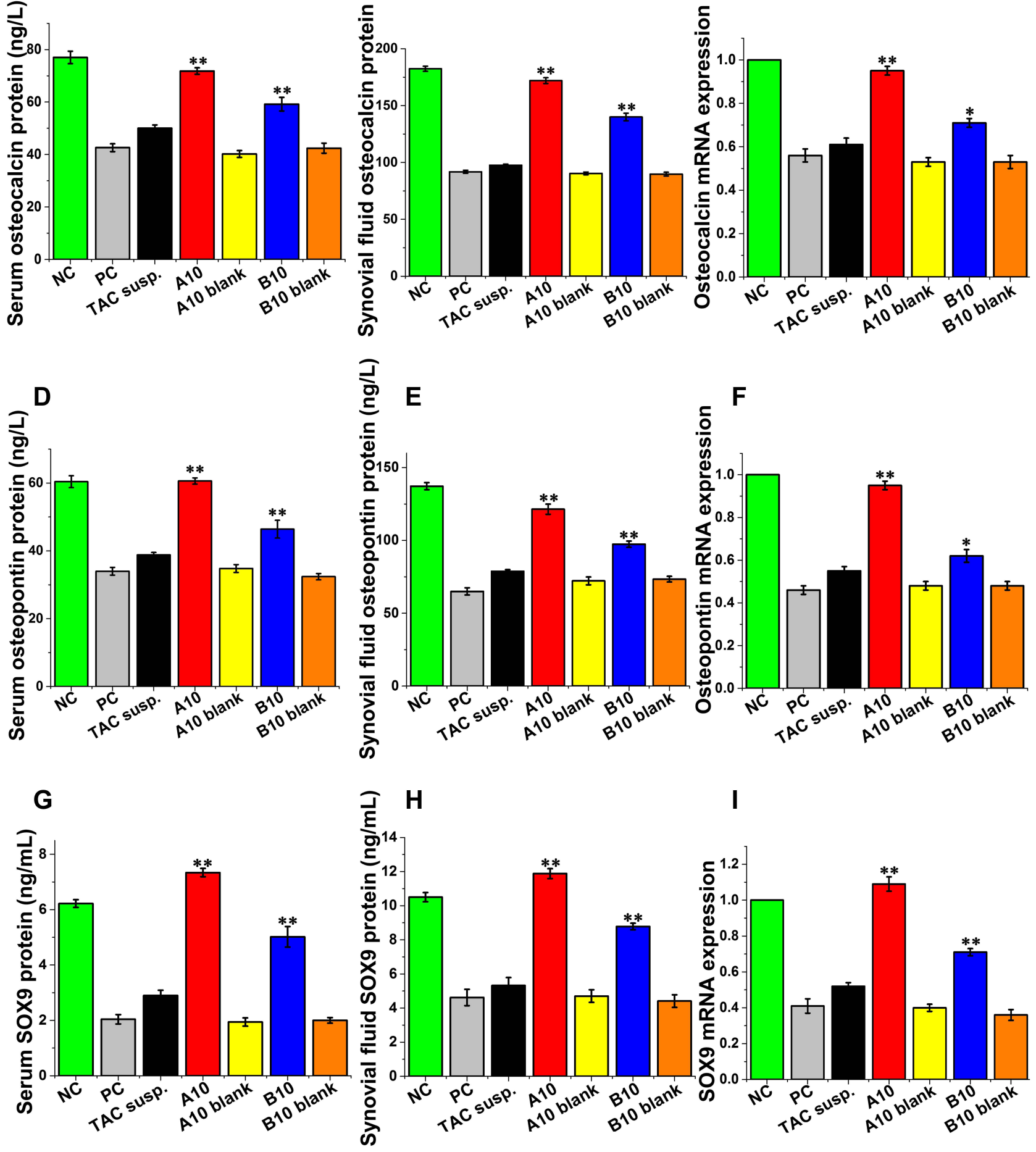

Figure 9 Concentration of bone and cartilage formation proteins: Level of osteocalcin protein in (A) serum, (B) synovial fluid and (C) its mRNA expression. Level of osteopontin protein in (D) serum, (E) synovial fluid and (F) its mRNA expression. level of SOX9 chondrogenic protein in (G) serum, $(\mathbf{H})$ synovial fluid and $(\mathbf{I})$ mRNA expression of SOX 9 chondrogenic protein. [* P 0.05 vs PC, ** P $\leq 0.05$ vs PC and TAC groups, mean \pm SEM, $n=5]$.

Abbreviations: NC, negative control; PC, positive control. 
with the group receiving $\mathbf{A 1 0}$ showing the greatest decrease in paw thickness with $\%$ reduction of $52.5 \% \pm 2.38$ followed by B10 group with \% reduction of $48.15 \% \pm 3.17$.

\section{Determination of Inflammatory Biomarkers}

The pathogenesis of rheumatoid arthritis is a complex process involving several humoral and cellular pathways where immune complex formation, vascular reactions, and infiltration of lymphocytes into the synovium occur. Consequently, proinflammatory mediators such as IL-6 (interleukin 6) are released to increase inflammation and destruction in the synovium. IL-6 plays a pivotal role in the pathophysiology and progression of RA as it promotes synovitis and joint destruction, stimulates pannus proliferation, and participates in developing systemic symptoms of RA.${ }^{47}$ Being abundant in the serum and synovial fluids of AIA model, IL-6 was a target to determine the severity and progression of arthritis. The serum levels of IL-6 are shown in Figure 8A. A significant reduction $(\mathrm{P}<0.05)$ in the level of IL-6 was observed at the end of the treatment period with TAC@MD$\alpha$-TOC nano-micelles when compared with positive control. On the other hand, groups that received blank micelles showed no effect on the elevated serum levels of IL-6.

Synovial fluid levels of IL-6 were also measured (Figure 8B) indicating almost the same pattern observed with the serum levels. Nano-micelles-treated groups had the maximum reduction in IL-6 synovial levels when compared with other groups. Also, blank micelles showed no influence on the synovial IL-6 levels.

TNF- $\alpha$ is also one of the key cytokine molecules associated with inflammation in RA. It enhances the proliferation of macrophages, activated T-cell and B-cell besides playing an important role in the formation of other inflammatory cytokines and joint destruction. ${ }^{48}$ As shown in Figure $8 \mathrm{C}$, TNF- $\alpha$ expression levels in serum were significantly inhibited in TAC@MD- $\alpha$-TOC treated groups when compared with the positive control. Administration of blank micelles also had no effect on serum TNF- $\alpha$ levels. TNF- $\alpha$ expression levels measured in the rats' synovial fluid showed a significant decrease $(\mathrm{P}<0.05)$ in the nano-micelles-treated groups when compared with positive control (Figure 8D).

The significant reduction in the serum and synovial levels of proinflammatory mediators (IL-6 and TNF- $\alpha$ ) in rats treated with the TAC@MD- $\alpha$-TOC nano-micelles compared with other groups could be attributed to the increased solubility of TAC in MD- $\alpha$-TOC nano-micelles besides the unique antioxidant activities of tailored bioconjugates. ${ }^{12}$ The efficient encapsulation of TAC within the nano-micellar core also played an essential role as it limited the release of drug in blood thereby reducing its binding to plasma components and increasing pharmacologically active portion of TAC in the arthritic tissue thus enhancing its efficacy. On the other hand, free TAC has high protein binding property to blood and plasma components making the unbound or pharmacologically active fraction of $\sim 1-2 \% .{ }^{37}$ Furthermore, the inflammatory nature of rheumatoid arthritis tissue with the highly permeable synovial vasculature along with the strong phagocytic capacity of activated synoviocytes during inflammatory conditions would allow the passage and sequestration of TAC-loaded micelles. This effect is known as "ELVIS" (extravasation through leaky vasculature and subsequent inflammatory cell-mediated sequestration) which could be responsible for the superior antirheumatic effect accompanied with MD- $\alpha$-TOC micelles. ${ }^{49}$ Moreover, the negative charge ( -20 to -22 $\mathrm{mv}$ ) on the surface of nano-micelles played a role in their accumulation in synovial fluid as reported previously by Ren et al. ${ }^{49}$ who attributed the reason to the high possibility of interaction between nano-micelles and synoviocytes to the slightly negative surface charge. Slightly negative surface charge also gave the advantage of decreased opsonization of nano-micelles thus increasing their abundance in blood and subsequently the rheumatic tissue. $^{16}$

The $\mathrm{pH}$-dependent release of TAC from the nanomicelles and the favored release at acidic $\mathrm{pH}$ of RA tissue played a beneficial role as it could account for the superiority of nano-micelles in alleviating RA inflammatory symptoms. Accordingly, TAC@MD- $\alpha$-TOC nano-micelles offered an enhanced TAC efficacy with apparent reduction in serum and synovial fluid levels of inflammatory mediators.

When comparing A10 and B10 groups, the B10 group had diminished serum IL-6 levels but to a lower extent compared with the A10 group. This could be attributed to the high hydrophobic interaction between high hydrophobic moieties in B10 polymer backbone and TAC decreasing drug release rate and slightly diminishing anti-arthritic effect with B10.

\section{Determination of Bone and Cartilage Formation Biomarkers}

Bone formation and bone resorption are two processes responsible for bone remodeling and are naturally present in equilibrium by utilizing both osteoclast and osteoblast 

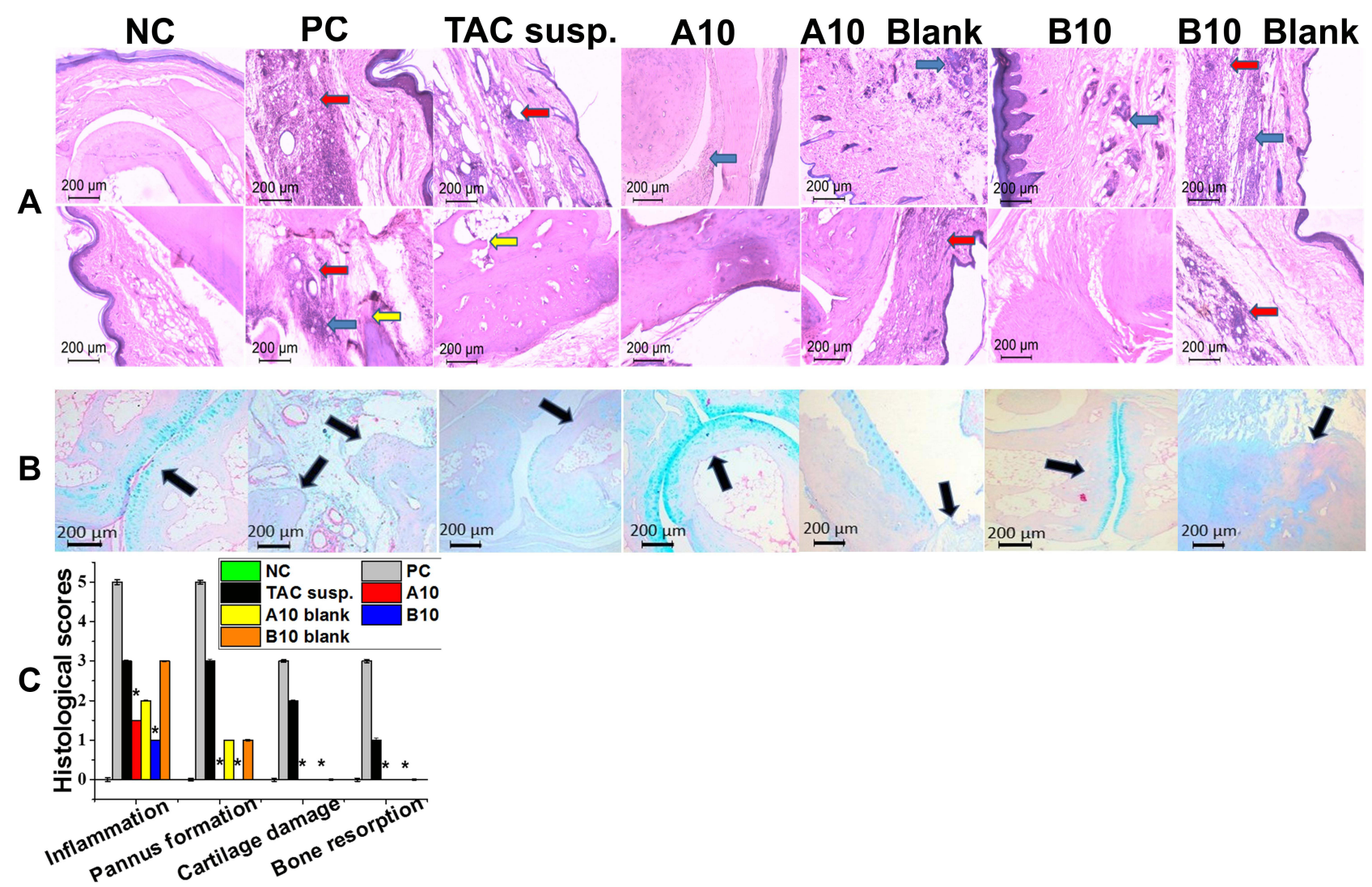

Figure 10 Histopathologic evaluation of AIA model paws. (A) H\&E staining, $\times 200$. (NC) Negative control group showing normal tissue, (PC) positive control group showing intense inflammatory infiltrate (blue arrow), extensive pannus formation (red arrow) and intense bone erosion (yellow arrow), (TAC susp.) group with moderate inflammatory infiltrate, moderate pannus formation (red arrow) and mild bone erosions (yellow arrow), (Al0) group with moderate inflammatory infiltrate (blue arrow), no pannus, normal cartilage, and bone, (AI0 blank) group showing moderate inflammatory infiltrate (blue arrow) with mild pannus formation (red arrow), (BI0) group with mild inflammatory infiltrate (blue arrow), no pannus, normal cartilage, and bone, (BI0) blank group showing severe inflammatory infiltrate (blue arrow) with mild pannus formation (red arrow). (B) Alcian blue staining, $\times 200$. (NC) with normal bone and cartilage (blue color, black arrow), (PC) with intense cartilage and bone erosion (black arrows), (Tac susp.)with mild cartilage erosion (black arrow), (Al0) with normal cartilage and bone (blue color, black arrow), (AI0 blank) with mild cartilage erosion and normal bone (black arrow), (BI0) with normal cartilage and bone (blue color, black arrow), (BIO blank) with mild cartilage erosion and normal bone (black arrow). (C) Histological scores of synovial inflammations, pannus formation, cartilage and bone erosion. [* P $\leq 0.05$ vs TAC groups, mean \pm SEM, $n=5]$.

activities. In RA condition, this equilibrium is shifted to bone resorption by osteoclasts leading to bone loss and joint damage. The abundance of osteoclast responsible for resorption of bone minerals along with impaired osteoblast signals are the main reasons beyond bone erosion in RA. Furthermore, presence of plenty of IL- 6 cytokine plays an important role indirectly on osteoclastogenesis. ${ }^{50,51}$

Osteocalcin and osteopontin are considered leading protein biomarkers in the bone formation process. They are produced by osteoblast and their serum presence marks bone formation process even at low rates. ${ }^{52,53}$ Serum and synovial fluid levels of osteocalcin and osteopontin in different treatment groups are illustrated in Figure 9A-F. Results reflected the unique effect of the nano-micelles in the bone formation process. Levels of serum osteocalcin and osteopontin were significantly $(\mathrm{P}<0.05)$ enhanced in TAC@MD- $\alpha$-TOC nano-micelles groups when compared with positive control and free TAC groups. For further affirmation of the effect on bone formation, both osteocalcin and osteopontin protein levels along with their mRNA expression in synovial fluid were measured. Synovial levels of osteocalcin and osteopontin increased significantly $(\mathrm{P}<0.05)$ with the nano-micelles treated groups compared with positive control and free TAC groups. mRNA expression levels of both osteocalcin and osteopontin were also determined to have an effect on the elevation of protein abundance. TAC@MD- $\alpha$-TOC nano-micelles-treated groups showed higher mRNA synovial levels when compared with TAC suspension (Figure 9C and F). This gave evidence that osteocalcin and osteopontin markers found in synovial fluid came from new cellular synthesis rather than from the release of bone matrix proteins during bone resorption. It was reported by Luo et al. ${ }^{54}$ and Kanda et al. ${ }^{55}$ that administration of TAC as an immunosuppressant drug had 
a negative impact on bone structure with increased bone resorption without significantly affecting bone formation. However, a recent study conducted by Nabavi et al. ${ }^{56}$ demonstrated preliminary evidence on the efficacy of TAC loaded hydrogel for the treatment of bone defects by decreasing levels of inflammatory mediators and improving osteogenic differentiation. According to our results, free TAC showed a minute effect on the bone formation biomarkers. However, this effect flourished upon the administration of TAC@MD- $\alpha$-TOC nano-micelles resulting in an increase in the bone formation biomarkers' abundance and expression. Although blank micelles had no effect on bone formation markers, native vitamin $\mathrm{E}$ was reported to have anti-osteoporotic properties by lowering the concentrations of free radicals that are responsible for stimulating bone resorption and suppressing bone formation. ${ }^{57}$ It has also been reported that administration of MD with calcium to postmenopausal women showed a better effect on markers of bone turnover. ${ }^{58}$ Those effects are concomitant with TAC sustained release in the arthritic tissue which lengthened the contact with osteocytes and might account for the observed enhanced expression of bone formation biomarkers that indicates promoted bone formation process.

RA is known as a bone and cartilage depleting disorder where the affected joints suffer from destruction of the cartilage, subchondral bone, and soft tissues. ${ }^{59}$ To measure the chondrogenic differentiation potential of prepared micelles, SOX9 protein was measured quantitatively in serum and synovial fluid. SOX9 is an important transcription protein that participates in cartilage development. It is active during chondrocyte differentiation and is highly demanded in chondrogenesis as it ensures chondrocyte lineage commitment, enhances cell survival, and transcriptionally activates the genes for many cartilage-specific structural components and regulatory factors. ${ }^{60}$ Figure $9 \mathrm{G}$ and $\mathrm{H}$ illustrate the impact of different treatments on serum and synovial levels of SOX9. A significant increase in serum and synovial levels of SOX9 protein accompanied treatment with TAC@MD- $\alpha-$ TOC nano-micelles. On the other hand, free TAC did not affect SOX9 levels giving a chondrogenic merit to the selection of MD- $\alpha$-TOC nano-micelles as TAC nanocarriers. Further evidence on the enhanced chondrogenic effect of TAC@MD- $\alpha$-TOC was provided by measuring the synovial mRNA expression levels of SOX9 protein (Figure 9I). It confirmed the preference of implementing TAC@MD- $\alpha-T O C$ as a successful delivery system for TAC in the management of rheumatoid arthritis.

\section{Histopathological Analysis for Arthritis}

Histopathological assessments for the paws and ankles of AIA treated rats are shown in Figure 10. At the end of the study, analyzing ankle inflammation, pannus infiltration, cartilage damage, and bone resorption clearly revealed evident protection from arthritis-altered parameters within TAC@MD- $\alpha$-TOC nano-micelles treated groups. Hematoxylin and eosin (H\&E) stained histological sections of joints are shown in Figure 10A where negative control group had normal tissue with absence of both inflammatory infiltrate and pannus. On the other hand, the positive control group showed intense inflammatory infiltrate with extensive pannus formation. Group treated with free TAC revealed moderate inflammatory infiltrate with moderate pannus formation. TAC@MD- $\alpha$-TOC (A10 and B10) treated groups showed a high protection impact on paws revealing moderate inflammatory infiltrate with absence of pannus. Moreover, blank nano-micellar treatment showed moderate inflammatory infiltrate with moderate pannus formation.

Cartilage of articular joints contains collagen type-II and plenty of proteoglycans. Wang et al. ${ }^{61}$ reported that the amount of proteoglycan over cartilage gets substantially reduced during arthritis conditions. Thus, in order to inspect proteoglycans in cartilage, Alcian blue staining was conducted (Figure 10B). Alcian blue dye is used to stain acidic mucopolysaccharides of proteoglycans which is indicative of cartilage damage in the articular joints. ${ }^{21}$ The negative control group (NC) had normal cartilage and bone tissues with intense alcian blue staining (shown as blue color) indicating abundance of proteoglycans. However, the positive control group (PC) exhibited obvious reduction in proteoglycan which indicated cartilage damage. Group treated with free TAC showed mild cartilage erosion. TAC@MD- $\alpha-T O C$ (A10 and B10) treated groups markedly reduced cartilage damage indicated by presence of higher proteoglycans (shown as blue color). Moreover, blank nano-micellar treatment showed mild cartilage and bone erosions.

Measured parameters were scored on a scale from 0 to 5 , with normal, minimal, mild, moderate, marked, and severe, respectively for $0,1,2,3,4$ and 5 . The scores of TAC@MD- $\alpha$-TOC nano-micelles were significantly $(\mathrm{P}<0.05)$ lower than those of free TAC (Figure 10C). Histopathological analysis confirmed to greater extent the treating properties of TAC@MD- $\alpha$-TOC nano-micelles on RA along with bone and cartilage regeneration criteria. 
According to the previous results of in vivo AIA experiment, TAC@MD- $\alpha$-TOC nano-micelles showed unique properties as an endeavor to manage RA and its combined bone and cartilage complications compared with free TAC suspension. Finally, and although obtained results were extraordinarily encouraging, further exploration and studies should be performed to understand the exact mechanisms for anti-rheumatic and bone regeneration activity of the prepared micelles.

\section{Conclusion}

In conclusion, tailored maltodextrin- $\alpha$-tocopherol amphiphilic polymers were successfully utilized to encapsulate TAC and employed as an anti-arthritic. Given the low aqueous solubility along with extensive systemic side effects, our TAC@MD- $\alpha$-TOC nano-micelles demonstrated the ability to improve TAC features both physically and biologically. Besides enhancing its safety profile without decreasing the therapeutic dose, TAC@MD- $\alpha$-TOC nano-micelles showed the property of introducing unique anti-arthritic and bone regeneration characteristics during arthritis assessment. This system, to our knowledge, is the first of its kind to combine antiarthritic, bone formation and chondrogenic differentiation activities without employing calcium salts. Preference of MD- $\alpha$-TOC also lies beyond the selection of naturally occurring substrates to prepare the amphiphilic copolymer (maltodextrin- $\alpha$-tocopherol) with high antioxidant activity, giving the advantage of combining safety and augmenting drug efficacy. Further investigations are advised to study the detailed mechanism of our nanomicellar system.

\section{Abbreviations}

TAC, Tacrolimus; MD- $\alpha$-TOC, maltodextrin- $\alpha$-tocopherol bioconjugate; TAC@MD- $\alpha$-TOC, Tacrolimus loadedmaltodextrin- $\alpha$-tocopherol nanomicelles; CFA, complete Freund's adjuvant; DLS, dynamic light scattering; DD, degree of derivatization; PBS, phosphate buffered saline.

\section{Acknowledgment}

This work was supported by the Faculty of Pharmacy, Alexandria University, ATRF committee [Grant ID: ATRF-0218006].

\section{Disclosure}

The authors report no conflicts of interest in this work.

\section{References}

1. McInnes IB, Schett G. The pathogenesis of rheumatoid arthritis. $N$ Engl J Med. 2011;365(23):2205-2219. doi:10.1056/NEJMra1004965

2. Zhang L, Chang J, Zhao Y, et al. Fabrication of a triptolide-loaded and poly- $\gamma$-glutamic acid-based amphiphilic nanoparticle for the treatment of rheumatoid arthritis. Int $J$ Nanomedicine. 2018;13:2051.

doi: 10 .

2147/IJN.S151233

3. Pandey S, Kumar V, Leekha A, et al. Co-delivery of teriflunomide and methotrexate from hydroxyapatite nanoparticles for the treatment of rheumatoid arthritis: in vitro characterization, pharmacodynamic and biochemical investigations. Pharm Res. 2018;35(11):201. doi:10.1007/s11095-018-2478-2

4. Wu $\mathrm{H}$, Wang $\mathrm{K}$, Wang $\mathrm{H}$, et al. Novel self-assembled tacrolimus nanoparticles cross-linking thermosensitive hydrogels for local rheumatoid arthritis therapy. Colloids Surf B Biointerfaces. 2017;14 9:97-104. doi:10.1016/j.colsurfb.2016.10.013

5. Byeon HJ, Lee C, Lee S, et al. Pharmaceutical potential of tacrolimus-loaded albumin nanoparticles having targetability to rheumatoid arthritis tissues. Int $J$ Pharm. 2016;497(1-2):268-276. doi:10.1016/j.ijpharm.2015.12.004

6. Thipparaboina R, Chavan RB, Kumar D, Modugula S, Shastri NR. Micellar carriers for the delivery of multiple therapeutic agents. Colloids Surf B Biointerfaces. 2015;135:291-308. doi:10.1016/j. colsurfb.2015.07.046

7. Shariatinia Z. Biopolymeric nanocomposites in drug delivery. $A d v$ Biopolymeric Sys Drug Delivery. 2020;233-290.

8. Cagel M, Tesan FC, Bernabeu E, et al. Polymeric mixed micelles as nanomedicines: achievements and perspectives. Eur $J$ Pharm Biopharm. 2017;113:211-228. doi:10.1016/j.ejpb.2016.12.019

9. Wang Q, Jiang $\mathrm{H}$, Li Y, et al. Targeting NF-kB signaling with polymeric hybrid micelles that co-deliver siRNA and dexamethasone for arthritis therapy. Biomaterials. 2017;122:10-22. doi:10.1016/j. biomaterials.2017.01.008

10. Wilson DR, Zhang N, Silvers AL, Forstner MB, Bader RA. Synthesis and evaluation of cyclosporine A-loaded polysialic acid-polycaprolactone micelles for rheumatoid arthritis. Eur J Pharm Sci. 2014;51:146-156. doi:10.1016/j.ejps.2013.09.013

11. Zhang JX, Yan MQ, Li XH, et al. Local delivery of indomethacin to arthritis-bearing rats through polymeric micelles based on amphiphilic polyphosphazenes. Pharm Res. 2007;24(10):1944-1953. doi:10. 1007/s11095-007-9322-4

12. Helal HM, Samy WM, El-Fakharany EM, Kamoun EA, Mortada SM, Sallam MA. Maltodextrin- $\alpha$-tocopherol conjugates of vitamin E: influence of degree of derivatization on physicochemical properties and biological evaluation. J Drug Deliv Sci Technol. 2020;102097. doi:10.1016/j.jddst.2020.102097

13. Tripodo G, Pasut G, Trapani A, et al. Inulin-d- $\alpha$-tocopherol succinate (INVITE) nanomicelles as a platform for effective intravenous administration of curcumin. Biomacromolecules. 2015;16(2):5 50-557. doi:10.1021/bm501616e

14. Helal HM, Mortada SM, Sallam MA. Paliperidone-loaded nanolipomer system for sustained delivery and enhanced intestinal permeation: superiority to polymeric and solid lipid nanoparticles. AAPS PharmSciTech. 2017;18(6):1946-1959. doi:10.1208/s12249-016-0657-1

15. Sallam MA, Helal HM, Mortada SM. Rationally designed nanocarriers for intranasaltherapy of allergic rhinitis: influence of carrier type on in vivo nasal deposition. Int $J$ Nanomedicine. 2016;11:2345. doi:10.2147/IJN.S98547

16. Anwar DM, Khattab SN, Helmy MW, et al. Lactobionic/folate dual-targeted amphiphilic maltodextrin-based micelles for targeted codelivery of sulfasalazine and resveratrol to hepatocellular carcinoma. Bioconjug Chem. 2018;29(9):3026-3041. doi:10.1021/ acs.bioconjchem. 8 b00428 
17. Li L, Tan YB. Preparation and properties of mixed micelles made of Pluronic polymer and PEG-PE. J Colloid Interface Sci. 2008;317 (1):326-331. doi:10.1016/j.jcis.2007.09.053

18. Wang J, Ma W, Guo Q, et al. The effect of dual-functional hyaluronic acid-vitamin E succinate micelles on targeting delivery of doxorubicin. Int J Nanomedicine. 2016;11:5851. doi:10.2147/IJN.S113882

19. Mosmann T. Rapid colorimetric assay for cellular growth and survival: application to proliferation and cytotoxicity assays. $J$ Immunol Methods. 1983;65(1-2):55-63. doi:10.1016/00221759(83)90303-4

20. Radwan -SE-S, Sokar MS, Abdelmonsif DA, El-Kamel AH. Mucopenetrating nanoparticles for enhancement of oral bioavailability of furosemide: in vitro and in vivo evaluation/sub-acute toxicity study. Int J Pharm. 2017;526(1-2):366-379. doi:10.1016/j.ijpharm. 2017.04.072

21. Ansari MM, Ahmad A, Kumar A, et al. Aminocellulose-graftedpolycaprolactone coated gelatin nanoparticles alleviate inflammation in rheumatoid arthritis: a combinational therapeutic approach. Carbohydr Polym. 2021;258:117600. doi:10.1016/j.carbpol.2020.117600

22. Shah SUA, Ashraf N, Soomro ZH, Shah MR, Kabir N, Simjee SU. The anti-arthritic and anti-oxidative effect of NBD (6-nitro-1, 3-benzodioxane) in adjuvant-induced arthritis (AIA) in rats. Inflamm Res. 2012;61(8):875-887. doi:10.1007/s00011-012-0480-4

23. Livak KJ, Schmittgen TD. Analysis of relative gene expression data using real-time quantitative PCR and the $2-\Delta \Delta \mathrm{CT}$ method. Methods. 2001;25(4):402-408. doi:10.1006/meth.2001.1262

24. Izumikawa M, Hayashi K, Polan MAA, Tang J, Saito T. Effects of amelogenin on proliferation, differentiation, and mineralization of rat bone marrow mesenchymal stem cells in vitro. ScientificWorldJournal. 2012;2012. doi:10.1100/2012/879731

25. Dai L, Zhang X, Hu X, Zhou C, Ao Y. Silencing of microRNA-101 prevents IL-1 $\beta$-induced extracellular matrix degradation in chondrocytes. Arthrit Res Ther. 2012;14(6):1-11. doi:10.1186/ar4114

26. Sallam MA, Prakash S, Krishnan V, Todorova K, Mandinova A, Mitragotri S. Hyaluronic Acid Conjugates of Vorinostat and Bexarotene for Treatment of Cutaneous Malignancies. Adv Ther. 2020;3(10):2000116. doi:10.1002/adtp.202000116

27. Sabra S, Abdelmoneem M, Abdelwakil M, et al. Self-assembled nanocarriers based on amphiphilic natural polymers for anti-cancer drug delivery applications. Curr Pharm Des. 2017;23 (35):5213-5229. doi:10.2174/1381612823666170526111029

28. Ö T, Çakır BA, Budama L, Hoda N. Determination of critical micelle concentration of polybutadiene-block-poly (ethyleneoxide) diblock copolymer by fluorescence spectroscopy and dynamic light scattering. J Mol Liq. 2013;177:40-43. doi:10.1016/j.molliq.2012. 10.013

29. Jones M-C, Leroux J-C. Polymeric micelles-a new generation of colloidal drug carriers. Eur $J$ Pharm Biopharm. 1999;48 (2):101-111. doi:10.1016/S0939-6411(99)00039-9

30. Wei W-H, Dong X-M, Liu C-G. In vitro investigation of self-assembled nanoparticles based on hyaluronic acid-deoxycholic acid conjugates for controlled release doxorubicin: effect of degree of substitution of deoxycholic acid. Int $J$ Mol Sci. 2015;16 (4):7195-7209. doi:10.3390/ijms16047195

31. Mitragotri S, Yoo J-W. Designing micro-and nanoparticles for treating rheumatoid arthritis. Arch Pharm Res. 2011;34(11):1887-1897. doi:10.1007/s12272-011-1109-9

32. Patel PV, Patel HK, Panchal SS, Mehta TA. Self-micro-emulsifying drug delivery system of tacrolimus: formulation, in vitro evaluation and stability studies. Int J Pharm Investig. 2013;3(2):95. doi:10.4103/ 2230-973X.114899

33. Yu D-H, Lu Q, Xie J, Fang C, Chen H-Z. Peptide-conjugated biodegradable nanoparticles as a carrier to target paclitaxel to tumor neovasculature. Biomaterials. 2010;31(8):2278-2292. doi:10.1016/j. biomaterials.2009.11.047
34. Zhang W, Shi Y, Chen Y, Hao J, Sha X, Fang X. The potential of Pluronic polymeric micelles encapsulated with paclitaxel for the treatment of melanoma using subcutaneous and pulmonary metastatic mice models. Biomaterials. 2011;32(25):5934-5944. doi:10.1016/j. biomaterials.2011.04.075

35. Ahamad N, Prabhakar A, Mehta S, et al. Trigger-responsive engineered nanocarriers and image-guided theranostics for Rheumatoid Arthritis. Nanoscale. 2020;12(24):12673-12697. doi:10.1039/D0NR01648A

36. Alam MM, Han HS, Sung S, et al. Endogenous inspired biomineral-installed hyaluronan nanoparticles as $\mathrm{pH}$-responsive carrier of methotrexate for rheumatoid arthritis. $J$ Controlled Release. 2017;252:62-72. doi:10.1016/j.jconrel.2017.03.012

37. Ghareeb M, Gohh RY, Akhlaghi F. Tacrolimus concentration in saliva of kidney transplant recipients: factors influencing the relationship with whole blood concentrations. Clin Pharmacokinet. 2018;57 (9):1199-1210. doi:10.1007/s40262-017-0626-1

38. Akar Y, Yucel G, Durukan AH, Yucel I, Arici G. Systemic toxicity of tacrolimus given by various routes and the response to dose reduction: laboratory Science. Clin Exp Ophthalmol. 2005;33(1):53-59. doi:10.1111/j.1442-9071.2005.00942.x

39. Neun BW, Ilinskaya AN, Dobrovolskaia MA. Updated Method for in vitro Analysis of Nanoparticle Hemolytic Properties. Characterization of Nanoparticles Intended for Drug Delivery. Springer; 2018:91-102.

40. Yoshikawa N, Yokota $\mathrm{T}$, Matsuo A, Matsumoto N, Iwakiri $\mathrm{T}$, Ikeda R. Role of FK506 Binding Protein on Tacrolimus Distribution in Red Blood Cells. Pharm Res. 2020;37(7):1-8. doi:10.1007/s11095-020-02875-z

41. Molaae N, Mosayebi G, Pishdadian A, Ejtehadifar M, Ganji A. Evaluating the proliferation of human peripheral blood mononuclear cells using MTT assay. Int J Basic Sci Med. 2017;2(1):25-28. doi:10.15171/ijbsm.2017.06

42. Ferjani H, El Arem A, Bouraoui A, et al. Protective effect of mycophenolate mofetil against nephrotoxicity and hepatotoxicity induced by tacrolimus in Wistar rats. J Physiol Biochem. 2016;72(2):133-144. doi: $10.1007 / \mathrm{s} 13105-015-0451-7$

43. Fung JJ. Tacrolimus and transplantation: a decade in review. Transplantation. 2004;77(9):S41-S43. doi:10.1097/01.TP.00001269 26.61434.A5

44. Liu Y, Han Y, Fang T, et al. Turning weakness into strength: albumin nanoparticle-redirected amphotericin B biodistribution for reducing nephrotoxicity and enhancing antifungal activity. $J$ Controlled Release. 2020;324:657-668. doi:10.1016/j.jconrel.2020.05.026

45. Du B, Yu M, Zheng J. Transport and interactions of nanoparticles in the kidneys. Nat Rev Materials. 2018;3(10):358-374. doi:10.1038/ s41578-018-0038-3

46. Firoozy M, Anarjan N. Preparation of maltodextrin stabilized $\alpha$ tocopherol nanoemulsions using solvent-displacement technique. Food Sci Technol Int. 2019;25(5):404-413. doi:10.1177/1082013219825893

47. Srirangan S, Choy EH. The role of interleukin 6 in the pathophysiology of rheumatoid arthritis. Ther Adv Musculoskelet Dis. 2010;2 (5):247-256. doi:10.1177/1759720X10378372

48. Vasanthi P, Nalini G, Rajasekhar G. Role of tumor necrosis factoralpha in rheumatoid arthritis: a review. APLAR J Rheum. 2007;10 (4):270-274. doi:10.1111/j.1479-8077.2007.00305.x

49. Ren H, He Y, Liang J, et al. Role of liposome size, surface charge, and PEGylation on rheumatoid arthritis targeting therapy. ACS Appl Mater Interfaces. 2019;11(22):20304-20315. doi:10.1021/acsami.8b22693

50. Le Goff B, Blanchard F, Berthelot J-M, Heymann D, Maugars Y. Role for interleukin-6 in structural joint damage and systemic bone loss in rheumatoid arthritis. Joint Bone Spine. 2010;77(3):201-205. doi:10.1016/j.jbspin.2010.03.002

51. Pan W, Dai C, Li Y, et al. PRP-chitosan thermoresponsive hydrogel combined with black phosphorus nanosheets as injectable biomaterial for biotherapy and phototherapy treatment of rheumatoid arthritis. Biomaterials. 2020;239:119851. doi:10.1016/j.biomaterials.2020.119851 
52. Cundy T, Reid IR, Grey A. CHAPTER 31 - Metabolic bone disease. In: Marshall WJ, Lapsley M, Day AP, Ayling RM, editors. Clinical Biochemistry: Metabolic and Clinical Aspects. Third ed. Churchill Livingstone; 2014:604-635.

53. Neve A, Corrado A, Cantatore FP. Osteoblast physiology in normal and pathological conditions. Cell Tissue Res. 2011;343(2):289-302. doi:10.1007/s00441-010-1086-1

54. Luo L, Shi Y, Bai Y, et al. Impact of tacrolimus on bone metabolism after kidney transplantation. Int Immunopharmacol. 2012;13 (1):69-72. doi:10.1016/j.intimp.2012.03.019

55. Kanda J, Izumo N, Furukawa M, et al. Effects of the calcineurin inhibitors cyclosporine and tacrolimus on bone metabolism in rats. Biomed Res. 2018;39(3):131-139. doi:10.2220/biomedres.39.131

56. Nabavi MH, Salehi M, Ehterami A, et al. A collagen-based hydrogel containing tacrolimus for bone tissue engineering. Drug Deliv Transl Res. 2020;10(1):108-121. doi:10.1007/s13346-019-00666-7

57. Wong SK, Mohamad N-V, Ibrahim NI, Chin K-Y, Shuid AN, ImaNirwana S. The molecular mechanism of vitamin $\mathrm{E}$ as a bone-protecting agent: a review on current evidence. Int J Mol Sci. 2019;20(6):1453. doi:10.3390/ijms20061453
58. Slevin MM, Allsopp PJ, Magee PJ, et al. Supplementation with calcium and short-chain fructo-oligosaccharides affects markers of bone turnover but not bone mineral density in postmenopausal women. $J$ Nutr. 2014;144(3):297-304. doi:10.3945/jn.113.188144

59. Ostrowska M, Maśliński W, Prochorec-Sobieszek M, Nieciecki M, Sudoł-Szopińska I. Cartilage and bone damage in rheumatoid arthritis. Reumatologia. 2018;56(2):111. doi:10.5114/reum.2018.75 523

60. Lefebvre V, Dvir-Ginzberg M. SOX9 and the many facets of its regulation in the chondrocyte lineage. Connect Tissue Res. 2017;58 (1):2-14. doi:10.1080/03008207.2016.1183667

61. Wang Y, Newman MR, Ackun-Farmmer M, et al. Fracture-targeted delivery of $\beta$-catenin agonists via peptide-functionalized nanoparticles augments fracture healing. ACS Nano. 2017;11(9):9445-9458. doi:10.1021/acsnano.7b05103
International Journal of Nanomedicine

\section{Publish your work in this journal}

The International Journal of Nanomedicine is an international, peerreviewed journal focusing on the application of nanotechnology in diagnostics, therapeutics, and drug delivery systems throughout the biomedical field. This journal is indexed on PubMed Central, MedLine, CAS, SciSearch ${ }^{\mathbb{R}}$, Current Contents ${ }^{\mathbb{B}} /$ Clinical Medicine, $^{2}$
Dovepress

Journal Citation Reports/Science Edition, EMBase, Scopus and the Elsevier Bibliographic databases. The manuscript management system is completely online and includes a very quick and fair peer-review system, which is all easy to use. Visit http://www.dovepress.com/ testimonials.php to read real quotes from published authors. 